\title{
Counting the dimension of splines of mixed smoothness
}

\section{A general recipe, and its application to planar meshes of arbitrary topologies}

\section{Deepesh Toshniwal $^{1}$ (1) $\cdot$ Michael DiPasquale $^{2}$}

Received: 2 March 2020 / Accepted: 19 November 2020 /

Published online: 17 January 2021

(C) The Author(s) 2021

\begin{abstract}
In this paper, we study the dimension of bivariate polynomial splines of mixed smoothness on polygonal meshes. Here, "mixed smoothness" refers to the choice of different orders of smoothness across different edges of the mesh. To study the dimension of spaces of such splines, we use tools from homological algebra. These tools were first applied to the study of splines by Billera (Trans. Am. Math. Soc. 310(1), 325-340, 1988). Using them, estimation of the spline space dimension amounts to the study of the Billera-Schenck-Stillman complex for the spline space. In particular, when the homology in positions 1 and 0 of this complex is trivial, the dimension of the spline space can be computed combinatorially. We call such spline spaces "lower-acyclic." In this paper, starting from a spline space which is loweracyclic, we present sufficient conditions that ensure that the same will be true for the spline space obtained after relaxing the smoothness requirements across a subset of the mesh edges. This general recipe is applied in a specific setting: meshes of arbitrary topologies. We show how our results can be used to compute the dimensions of spline spaces on triangulations, polygonal meshes, and T-meshes with holes.
\end{abstract}

Keywords Splines · Polygonal meshes with holes $\cdot$ Spline dimension formulas · Mixed smoothness

Mathematics Subject Classification (2010) 13D02 · 41A15

Communicated by: Larry L. Schumaker

Deepesh Toshniwal

d.toshniwal@tudelft.nl

1 Delft Institute of Applied Mathematics, Delft University of Technology,

Delft 2628XE, The Netherlands

2 Department of Mathematics, Colorado State University, Fort Collins, CO, USA 


\section{Introduction}

Piecewise-polynomial functions called splines are foundational pillars that support modern computer-aided geometric design [1], numerical analysis [2], etc. These functions are defined on polyhedral partitions of $\mathbb{R}^{n}$. Their restriction to any polyhedron's interior is a polynomial, and these polynomial pieces are constrained to join with some desired smoothness across hyperplanes supporting the intersections of neighboring polyhedra. Here, we study bivariate spline spaces-i.e., $n=2-$ of mixed smoothness-i.e., different orders of smoothness constraints are imposed across different edges of the partition. From the perspective of approximation with splines, mixed smoothness is particularly interesting for capturing local, non-smooth (or even discontinuous) features in the target function, e.g., for shock-capturing in fluid dynamics, or for modeling smooth geometries with localized creases. In particular, we study how the dimension of such spline spaces can be computed.

Computing the dimension of spline spaces is a highly non-trivial task in general for splines in more than one variable. Initiated by Strang [3, 4], this is by now a classical topic in approximation theory and has been studied in a wide range of planar settings, e.g., on triangulations, polygonal meshes, and T-meshes [5-14]. Non-polynomial spline spaces have also been studied in the same vein, e.g., [15].

In the present paper, instead of initiating the study of mixed-smoothness splines from scratch, we study them in relation to a proper subspace for which the dimensioncomputation problem is well-understood. Several conceptually similar approaches have been recently formulated, inspired by applications of splines in numerical analysis and geometric modeling. For instance, this approach was adopted to study splines on non-rectangular T-meshes in [16] (using Bernstein-Bézier methods); to study splines on locally subdivided simplicial meshes in [17]; to study splines with local polynomial-degree adaptivity in $[12,14]$; and to study mixed-smoothness splines on T-meshes in [18].

More specifically, we derive sufficient conditions that help describe mixedsmoothness spline spaces as lower-acyclic, i.e., as spaces for which the dimension can be computed combinatorially using only local geometric information. Working on a polygonal mesh in $\mathbb{R}^{2}$, we start from a spline space $\mathcal{R}^{r}$ whose members are constrained to be at least $\boldsymbol{r}(\tau)$ smooth across edge $\tau$ of the mesh. Then, given that $\mathcal{R}^{r}$ is lower-acyclic, we derive sufficient conditions for $\mathcal{R}^{s} \supseteq \mathcal{R}^{r}$ to be lower-acyclic, where $\boldsymbol{s}(\tau) \leqslant \boldsymbol{r}(\tau)$ for all edges $\tau$. In general, the former does not imply the latter [18]. We use methods from homological algebra to derive these results; see Section 3.

In order to examine the sufficient conditions in practice, we narrow our focus down to a specific application: dimension computation for spline spaces on meshes of arbitrary topologies; e.g., see Fig. 1. Such splines enable geometric modeling of and numerical analysis on arbitrary smooth surfaces [19], and are very useful in applications. We investigate the applications of our results to the following particular cases; see Section 4 for details:

- Total-degree splines on triangulations and polygonal meshes containing holes; 


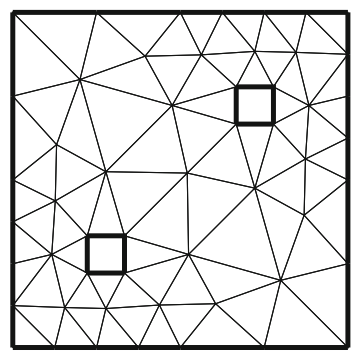

(a)

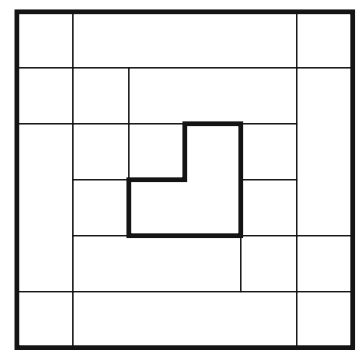

(b)

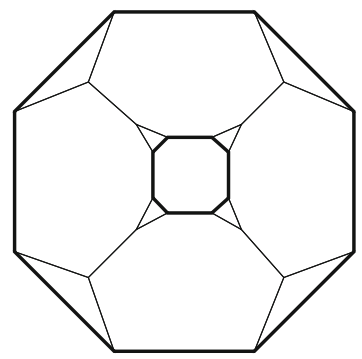

(c)

Fig. 1 We study the dimension of splines on polygonal meshes of arbitrary topologies, such as the ones shown above, in Section 4. Here, the mesh boundaries have been displayed in bold

- Mixed bi-degree splines on T-meshes containing holes.

In summary, the following are our main contributions in this paper. In Section 3, we derive a highly general dimension-computation framework with a plug-and-play nature; i.e., it can be combined with other approaches from the literature to compute dimensions of a wide variety of non-standard spline spaces. We illustrate its utility in Section 4 by applying it to the study of spline spaces defined on triangulations, polygonal meshes, and T-meshes. Doing so, we recover and extend results in several papers [16, 18, 20, 21]. For instance:

- In Section 4.1, we provide a new proof that the spline complex of degree $m$ and regularity $r$ on an arbitrary triangulation is exact when $m \geqslant 3 r+2$ [20], and provide better estimates for non-generic situations with low degree and high smoothness.

- We recover dimension formulas for $C^{r}$ splines for arbitrary polygonal meshes with holes $[10,11,21]$ for large enough degree $m$ in Section 4.2.

- In Section 4.3, we derive simple geometric conditions that allow a combinatorial dimension formula for mixed smoothness splines with non-uniformly chosen bi-degrees on T-meshes, thus extending the results from [14, 18]. Furthermore, we show how our results can be more broadly applied to study the dimension of splines on T-meshes with holes, while circumventing the need to compute dimensions of splines on non-rectangular T-meshes with boundary conditions [16].

Consequently, our work also demonstrates the uniform applicability of homological methods to the study of splines on various mesh types.

\section{Preliminaries: splines, meshes, and homology}

This section will introduce the relevant notation that we will use for working with polynomial splines on polygonal meshes. 


\subsection{Bivariate splines on planar meshes}

Definition 2.1 (Mesh) A mesh $\mathcal{T}$ of $\mathbb{R}^{2}$ is defined as:

- A finite collection $\mathcal{T}_{2}$ of polygons $\sigma$ that we consider as open sets of $\mathbb{R}^{2}$ having non-zero measure, called 2-cells or faces, together with

- A finite set $\mathcal{T}_{1}$ of closed segments $\tau$, called 1-cells, which are edges of the (closure of the) faces $\sigma \in \mathcal{T}_{2}$, and

- The set $\mathcal{T}_{0}$, of vertices $\gamma$, called 0 -cells, of the edges $\tau \in \mathcal{T}_{1}$,

such that the following properties are satisfied:

- $\sigma \in \mathcal{T}_{2} \Rightarrow$ the boundary of $\sigma$, denoted $\partial \sigma$, is a finite union of edges in $\mathcal{T}_{1}$,

- $\sigma, \sigma^{\prime} \in \mathcal{T}_{2} \Rightarrow \bar{\sigma} \cap \overline{\sigma^{\prime}}=\partial \sigma \cap \partial \sigma^{\prime}$, if non-empty, is a single edge in $\mathcal{T}_{1}$ or a single vertex in $\mathcal{T}_{0}$, and,

- $\tau, \tau^{\prime} \in \mathcal{T}_{1}$ with $\tau \neq \tau^{\prime} \Rightarrow \tau \cap \tau^{\prime} \in \mathcal{T}_{0}$,

where $\bar{\sigma}$ denotes the closure of the mesh face $\sigma$. The domain of the mesh is assumed to be connected and is defined as $\Omega:=\cup_{\sigma \in \mathcal{T}_{2}} \bar{\sigma} \subset \mathbb{R}^{2}$.

Edges of the mesh will be called interior edges if they intersect the interior of the domain of the mesh, $\stackrel{\circ}{\Omega}$. Otherwise, they will be called boundary edges. The set of interior edges will be denoted by $\stackrel{\circ}{\mathcal{T}}_{1}$. Similarly, if a vertex is in $\stackrel{\Omega}{\Omega}$ it will be called an interior vertex, and a boundary vertex otherwise. The set of interior vertices will be denoted by $\stackrel{\circ}{\mathcal{T}}_{0}$.

The first ingredient we need for defining polynomial splines on $\mathcal{T}$ are vector spaces of polynomials attached to each face of the mesh. More precisely, to each face $\sigma$ of the mesh, we will assign a vector space of (total degree or bi-degree) polynomials denoted by $\mathcal{P}_{\sigma}$ :

$$
\boldsymbol{m}: \sigma \mapsto \mathcal{P}_{\sigma} .
$$

If the closures of faces $\sigma$ and $\sigma^{\prime}$ have non-empty intersection, then we will assume that

$$
\mathcal{P}_{\sigma}+\mathcal{P}_{\sigma^{\prime}}=\mathcal{P}_{\sigma} \text { or } \mathcal{P}_{\sigma^{\prime}} .
$$

Then, we can use $\mathcal{P}_{\sigma}$ to assign vector spaces of polynomials to the edges and vertices of $\mathcal{T}$. Denoting these by $\mathcal{P}_{\tau}$ and $\mathcal{P}_{\gamma}$, respectively, for $\tau \in \mathcal{T}_{1}$ and $\gamma \in \mathcal{T}_{0}$, we define them as follows:

$$
\mathcal{P}_{\tau}:=\sum_{\bar{\sigma} \supset \tau} \mathcal{P}_{\sigma}, \quad \mathcal{P}_{\gamma}:=\sum_{\bar{\sigma} \ni \gamma} \mathcal{P}_{\sigma} .
$$

The above assignment of vector spaces to faces, edges, and vertices of $\mathcal{T}$ will be assumed to be fixed throughout this document.

The second and final ingredient that we need for defining splines on $\mathcal{T}$ is a smoothness distribution on its edges. The objective of this paper is to study how the dimension of the space of splines on $\mathcal{T}$ (which will be defined shortly) changes with this smoothness distribution. 
Definition 2.2 (Smoothness distribution) The map $\boldsymbol{r}: \mathcal{T}_{1} \rightarrow \mathbb{Z}_{\geqslant-1}$ is called a smoothness distribution if $\boldsymbol{r}(\tau)=-1$ for all $\tau \notin \stackrel{\circ}{\mathcal{T}}_{1}$.

Using this notation, we can now define the spline space $\mathcal{R}^{r}$ that forms the object of our study. From the following definition and the definition of $\boldsymbol{r}$, it will be clear that we are interested in obtaining highly local control over the smoothness of splines in $\mathcal{R}^{r}$, a feature that is missing from most of the existing literature. This problem has been addressed in a recent paper [18], but in a restricted setting where it is assumed that (a) $\mathcal{T}$ is a T-mesh and (b) $\mathcal{P}_{\sigma}=\mathcal{P}_{\sigma^{\prime}}$ for any $\sigma, \sigma^{\prime} \in \mathcal{T}_{2}$.

Definition 2.3 (Spline space) The spline space $\mathcal{R}^{r}$ is defined as:

$$
\begin{aligned}
& \mathcal{R}^{r}:=\mathcal{R}_{\boldsymbol{m}}^{\boldsymbol{r}}(\mathcal{T}):=\left\{f:\left.\forall \sigma \in \mathcal{T}_{2} \quad f\right|_{\sigma} \in \mathcal{P}_{\sigma},\right. \\
& \left.\forall \tau \in \stackrel{\circ}{\mathcal{T}}_{1} f \text { is } C^{r(\tau)} \text { smooth across } \tau\right\} .
\end{aligned}
$$

From the above definition, the pieces of all splines in $\mathcal{R}^{r}$ are constrained to meet with smoothness $\boldsymbol{r}(\tau)$ at an interior edge $\tau$. We will use the following algebraic characterization of smoothness in this document (for a proof see [7] or [22]).

Lemma 2.4 For $\sigma, \sigma^{\prime} \in \mathcal{T}_{2}$, let $\bar{\sigma} \cap \overline{\sigma^{\prime}}=\tau \in \stackrel{\circ}{\mathcal{T}}_{1}$, and consider a piecewise polynomial function equalling $p$ and $q$ on $\sigma$ and $\sigma^{\prime}$, respectively. Then, this piecewise polynomial function is at least $r$ times continuously differentiable across $\tau$ if and only if

$$
\ell_{\tau}^{r+1} \mid p-q
$$

where $\ell_{\tau}$ is a non-zero linear polynomial vanishing on $\tau$.

In line with the above characterization and for each interior edge $\tau$, we define $\mathfrak{I}_{\tau}^{r}$ to be the vector subspace of $\mathcal{P}_{\tau}$ that contains all polynomial multiples of $\ell_{\tau}^{r(\tau)+1}$; when $\boldsymbol{r}(\tau)=-1, \mathfrak{I}_{\tau}^{\boldsymbol{r}}$ is simply defined to be $\mathcal{P}_{\tau}$. Similarly, for each interior vertex $\gamma$, we define $\mathfrak{I}_{\gamma}^{r}:=\sum_{\tau \ni \gamma} \mathfrak{I}_{\tau}^{r}$. Note that, once specified, $\boldsymbol{m}$ is assumed to be fixed; here we are only interested in how the spline space changes with the smoothness distribution. Thus, we have suppressed the dependence of the different spaces on $\boldsymbol{m}$ to simplify the reading (and writing) of the text.

\subsection{Topological chain complexes}

Any spline $f \in \mathcal{R}^{r}$ is a piecewise polynomial function on $\mathcal{T}$. We can explicitly refer to its piecewise polynomial nature by equivalently expressing it $\sum_{\sigma}[\sigma] f_{\sigma}$ with $f_{\sigma}:=\left.f\right|_{\sigma}$. This notation makes it clear that the polynomial $f_{\sigma}$ is attached to the face $\sigma$ of $\mathcal{T}$. Using this notation and Lemma 2.4, the spline space $\mathcal{R}^{r}$ can be equivalently expressed as the kernel of the map $\bar{\partial}$, where

$$
\bar{\partial}: \underset{\sigma \in \mathcal{T}_{2}}{\bigoplus_{2}}[\sigma] \mathcal{P}_{\sigma} \rightarrow \underset{\tau \in \mathcal{T}_{1}}{\oplus_{\sigma}}[\tau] \mathcal{P}_{\tau} / \mathfrak{I}_{\tau}^{r}
$$


is defined by composing the cellular chain map $\partial$ (with local coefficients) relative to the boundary of $\mathcal{T}$, namely

$$
\partial: \underset{\sigma \in \mathcal{T}_{2}}{\bigoplus}[\sigma] \mathcal{P}_{\sigma} \rightarrow \underset{\tau \in \mathcal{T}_{1}}{\oplus_{0}}[\tau] \mathcal{P}_{\tau},
$$

with the natural quotient map $q: \underset{\tau \in \mathcal{T}_{1}}{\oplus}[\tau] \mathcal{P}_{\tau} \rightarrow \underset{\tau \in \mathcal{T}_{1}}{\oplus}[\tau] \mathcal{P}_{\tau} / \mathfrak{I}_{\tau}^{r}$.

We briefly illustrate the cellular chain map relative to the boundary in Example 2.5 and direct the reader to [23] for additional details on cellular and simplicial homology.

Example 2.5 Consider the mesh $\mathcal{T}$ shown in Fig. 2 with three interior edges, three boundary edges, and a single interior vertex. With orientations on edges as indicated in Fig. 2 and with each face oriented in a counterclockwise manner, the usual cellular chain map from faces to edges is given by:

$\left[\sigma_{1}\right] \mapsto\left[\tau_{4}\right]-\left[\tau_{1}\right]+\left[\tau_{3}\right], \quad\left[\sigma_{2}\right] \mapsto\left[\tau_{1}\right]+\left[\tau_{5}\right]-\left[\tau_{2}\right], \quad\left[\sigma_{3}\right] \mapsto\left[\tau_{2}\right]+\left[\tau_{6}\right]-\left[\tau_{3}\right]$.

The chain maps we consider, which we write as $\partial$, are relative to the mesh-boundary, i.e., modulo all (formal sums of) boundary edges and vertices. Hence, our chain map from faces to interior edges is given by:

$$
\partial\left(\left[\sigma_{1}\right]\right)=-\left[\tau_{1}\right]+\left[\tau_{3}\right], \quad \partial\left(\left[\sigma_{2}\right]\right)=\left[\tau_{1}\right]-\left[\tau_{2}\right], \quad \partial\left(\left[\sigma_{3}\right]\right)=\left[\tau_{2}\right]-\left[\tau_{3}\right] .
$$

We extend this linearly to $\left[\sigma_{1}\right] \mathcal{P}_{\sigma_{1}} \oplus\left[\sigma_{2}\right] \mathcal{P}_{\sigma_{2}} \oplus\left[\sigma_{3}\right] \mathcal{P}_{\sigma_{3}}$. Representing $f=\left[\sigma_{1}\right] f_{\sigma_{1}}+$ $\left[\sigma_{2}\right] f_{\sigma_{2}}+\left[\sigma_{3}\right] f_{\sigma_{3}}$ as a column vector, the map $\partial: \underset{\sigma \in \mathcal{T}_{2}}{\bigoplus}[\sigma] \mathcal{P}_{\sigma} \rightarrow \underset{\tau \in \dot{\mathcal{T}}_{1}}{\oplus_{0}}[\tau] \mathcal{P}_{\tau}$ is given by multiplication by the matrix:

$$
\left[\begin{array}{ccc}
-1 & 1 & 0 \\
0 & -1 & 1 \\
1 & 0 & -1
\end{array}\right],
$$

with columns corresponding to $\sigma_{1}, \sigma_{2}$, and $\sigma_{3}$ and rows to $\tau_{1}, \tau_{2}$, and $\tau_{3}$.

Similarly, our chain map from interior edges to interior vertices is given by

$$
\partial\left(\left[\tau_{1}\right]\right)=-\left[\gamma_{1}\right], \quad \partial\left(\left[\tau_{2}\right]\right)=-\left[\gamma_{1}\right], \quad \partial\left(\left[\tau_{3}\right]\right)=-\left[\gamma_{1}\right] .
$$

We again extend this linearly to $\left[\tau_{1}\right] \mathcal{P}_{\tau_{1}} \oplus\left[\tau_{2}\right] \mathcal{P}_{\tau_{2}} \oplus\left[\tau_{3}\right] \mathcal{P}_{\tau_{3}}$. Representing $f=$ $\left[\tau_{1}\right] f_{\tau_{1}}+\left[\tau_{2}\right] f_{\tau_{2}}+\left[\tau_{3}\right] f_{\tau_{3}}$ as a column vector, the map $\partial: \underset{\tau \in \mathcal{T}_{1}}{\oplus}[\tau] \mathcal{P}_{\tau} \rightarrow \underset{\gamma \in \mathcal{T}_{0}}{\oplus}[\gamma] \mathcal{P}_{\gamma}$

Fig. 2 The mesh in Example 2.5

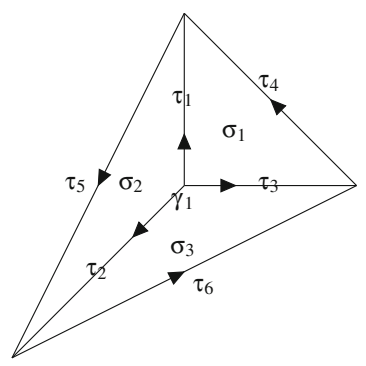


is given by multiplication by the matrix:

$$
\left[\begin{array}{lll}
-1 & -1-1
\end{array}\right],
$$

with columns corresponding to $\tau_{1}, \tau_{2}$, and $\tau_{3}$ and the row to $\gamma_{1}$.

Since the spline space $\mathcal{R}^{r}$ is the kernel of the map $\bar{\partial}$ in Eq. 2.3, it can be interpreted as the top homology of a suitably defined chain complex $Q^{r}$,

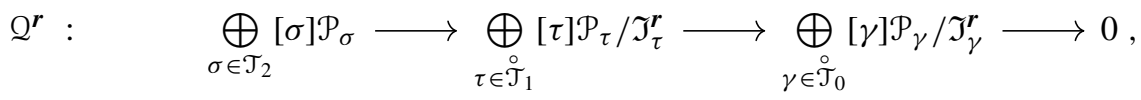

where the second map is induced from the usual cellular chain map from edges to vertices. We call $Q^{r}$ the Billera-Schenck-Stillman complex (abbreviated as BSS). The BSS was first introduced in [7,9], and was first studied for purposes of local degree elevation in $[12,14]$; additional details on its construction may be found in these references. As in $[7,9,13]$, we will study $Q^{r}$ using the following short exact sequence of chain complexes:

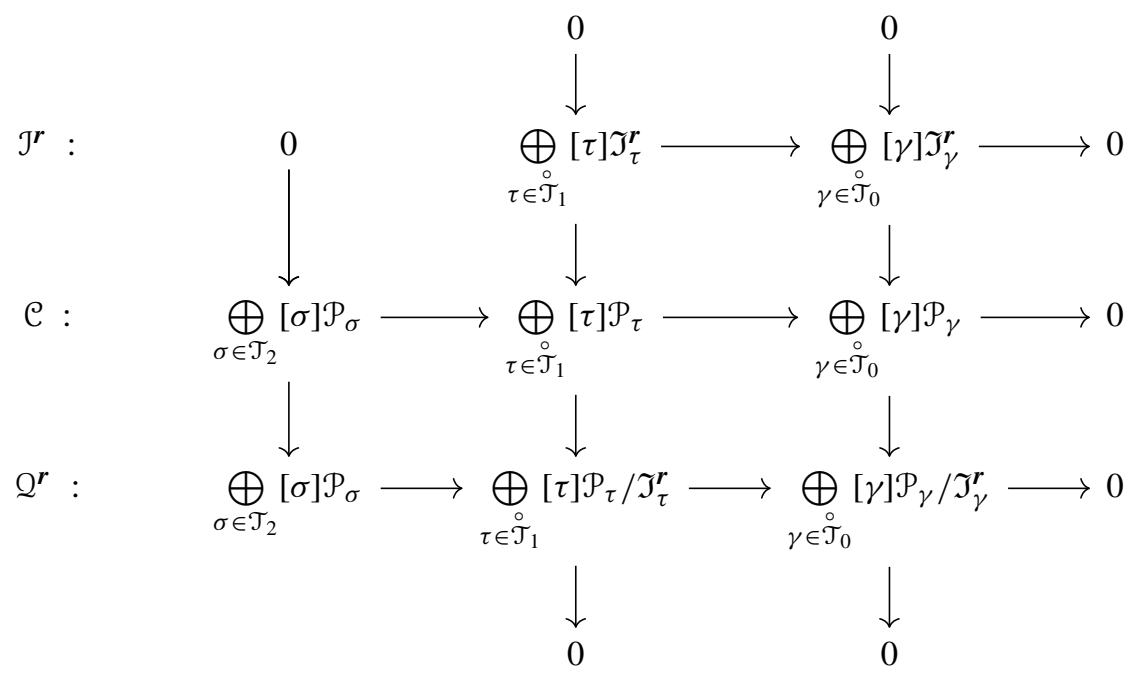

It should be emphasized that all objects in the above diagram are vector spaces and not modules; recall that we have merely suppressed their dependence on the chosen degree distribution $\boldsymbol{m}$.

Definition 2.6 (Lower-acyclicity of BSS) The complex $Q^{r}$ will be called loweracyclic if its homologies in positions 1 and 0 are trivial, i.e., if $H_{1}\left(Q^{r}\right)=0=$ $H_{0}\left(Q^{r}\right)$.

Lower-acyclicity of the BSS is interesting precisely because it is a sufficient condition for ensuring that the dimension of $\mathcal{R}^{r}$ can be computed using only local combinatorial data, with the computation being unaffected by the global geometry of $\mathcal{T}$. 
Theorem 2.7 If $Q^{r}$ is lower-acyclic, then the dimension of $\mathcal{R}^{r}$ can be combinatorially computed,

$$
\operatorname{dim}\left(\mathcal{R}^{r}\right)=\chi\left(Q^{r}\right),
$$

where $\chi\left(Q^{r}\right)$ is the Euler characteristic of the complex $Q^{r}$. Moreover,

$$
H_{0}\left(\mathcal{J}^{r}\right) \cong H_{0}(\mathcal{C}) \text {. }
$$

Proof The first claim follows from the definition of the Euler characteristic of $Q^{r}$ :

$$
\begin{aligned}
\chi\left(Q^{r}\right) & =\operatorname{dim}\left(Q_{2}^{r}\right)-\operatorname{dim}\left(Q_{1}^{r}\right)+\operatorname{dim}\left(Q_{0}^{r}\right), \\
& =\operatorname{dim}\left(H_{2}\left(Q^{r}\right)\right)-\operatorname{dim}\left(H_{1}\left(Q^{r}\right)\right)+\operatorname{dim}\left(H_{0}\left(Q^{r}\right)\right), \\
& =\operatorname{dim}\left(\mathcal{R}^{r}\right)-\operatorname{dim}\left(H_{1}\left(Q^{r}\right)\right)+\operatorname{dim}\left(H_{0}\left(Q^{r}\right)\right) .
\end{aligned}
$$

The second part of the claim follows from the long exact sequence of homologies implied by the short exact sequence of chain complexes in Equation (2.4),

$$
\cdots \longrightarrow H_{1}(\mathrm{C}) \longrightarrow H_{1}\left(Q^{r}\right) \longrightarrow H_{0}\left(\mathcal{J}^{r}\right) \longrightarrow H_{0}(\mathrm{C}) \longrightarrow H_{0}\left(Q^{r}\right) \longrightarrow 0 .
$$

Remark 2.8 We describe the meaning of Theorem 2.7 in the case of uniform degree and smoothness on planar triangulations. Suppose $\mathcal{T}$ is a simply connected planar triangulation, $\boldsymbol{m}$ assigns to each face the vector space of polynomials with total degree $m$, and $\boldsymbol{r}$ assigns to each interior edge the same fixed non-negative integer $r$. Under these assumptions, the Euler characteristic $\chi\left(Q^{r}\right)$ of the Billera-Schenck-Stillman chain complex is precisely the lower bound of Schumaker [5], as is shown in [9]. The main result of [24] is that Schumaker's lower bound gives the exact dimension of the spline space over a generic triangulation when $m \geq 3 r+1$. Translated into our terminology, this is the same as saying that $Q^{r}$ is lower-acyclic for $m \geq 3 r+1$.

\section{Spline space $\mathcal{R}^{s} \supseteq \mathcal{R}^{r}$ of reduced regularity}

We present our main results in this section. In particular, we will relate the dimension of the spline space $\mathcal{R}^{r}$ to the dimension of a spline space $\mathcal{R}^{s}$ obtained by relaxing the regularity requirements. That is, for all interior edges $\tau$, it will be assumed that $\boldsymbol{s}(\tau) \leqslant \boldsymbol{r}(\tau)$. This relationship will be utilized to present sufficient conditions for lower-acyclicity of the BSS defined for $\mathcal{R}^{s}$.

For the spline space $\mathcal{R}^{s}$, let the first and last chain complexes in Equation (2.4) be denoted by $\mathcal{J}^{s}$ and $\mathcal{Q}^{s}$, respectively. Then, by definition of the smoothness distributions $\boldsymbol{r}$ and $\boldsymbol{s}$, we have the following inclusion map from $\mathcal{J}^{r}$ to $\mathcal{J}^{s}$,

$$
\mathrm{J}^{r} \rightarrow \mathrm{J}^{s} .
$$

Since both complexes are also included in the complex $\mathcal{C}$, we can build the following commuting diagram between two short exact sequences of chain complexes:

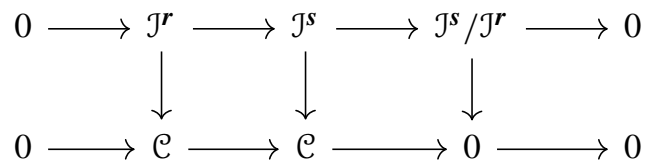


Proposition 3.1 If $H_{0}\left(\mathcal{J}^{s} / \mathcal{J}^{r}\right)=0$ and $Q^{r}$ is lower-acyclic, then the following hold:

$$
H_{0}\left(\mathcal{J}^{s}\right) \cong H_{0}(\mathcal{C}) \cong H_{0}\left(\mathcal{J}^{r}\right), \quad H_{0}\left(Q^{s}\right)=0 .
$$

Proof The diagram in Equation (3.1) implies the following commuting diagram that connects the long exact sequence of homologies for the two exact sequences of complexes:

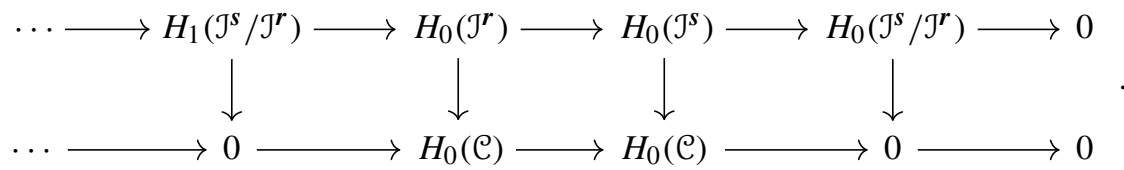

Then, from Theorem 2.7 , we know that $H_{0}(\mathcal{C}) \cong H_{0}\left(\mathcal{J}^{r}\right)$. Then, by an application of the Five lemma [23, 25], we obtain

$$
H_{0}\left(\mathcal{J}^{s}\right) \cong H_{0}(\mathcal{C})
$$

and the first part of the claim follows. The second part of the claim follows upon considering the long exact sequence of homologies implied by the following short exact sequence of chain complexes:

$$
\mathrm{J}^{s} \longrightarrow \mathrm{C} \longrightarrow \mathrm{Q}^{s}
$$

The tail-end of this long exact sequence is shown below and, using the first part of the claim, we see that $H_{0}\left(Q^{s}\right)$ must be trivial:

$$
\cdots \longrightarrow H_{0}\left(\mathcal{J}^{s}\right) \longrightarrow H_{0}(\mathrm{C}) \longrightarrow H_{0}\left(Q^{s}\right) \longrightarrow 0
$$

By focusing on the simpler object $H_{0}\left(\mathcal{J}^{s} / \mathcal{J}^{r}\right)$, see Lemma 3.4 at the end of this section, the previous result helps identify when $H_{0}\left(\mathcal{J}^{s}\right)$ will be isomorphic to $H_{0}(\mathcal{C})$. Using this, we can now present our main results: sufficient conditions for the loweracyclicity of $Q^{s}$. The results use the following commuting diagram of complexes:

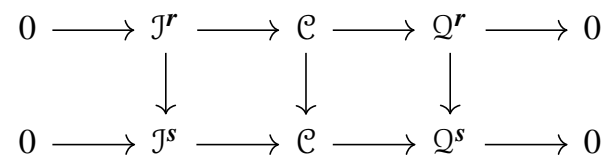

which is built using the inclusion map $\mathcal{C} \rightarrow \mathcal{C}$.

Proposition 3.2 If $H_{0}\left(\mathcal{J}^{s} / \mathcal{J}^{r}\right)=0$ and $Q^{r}$ is lower-acyclic, then

$$
H_{1}\left(Q^{s}\right)=0 .
$$

Proof The diagram in Equation (3.2) implies the following commuting diagram that connects the long exact sequence of homologies for the two exact sequences of complexes:

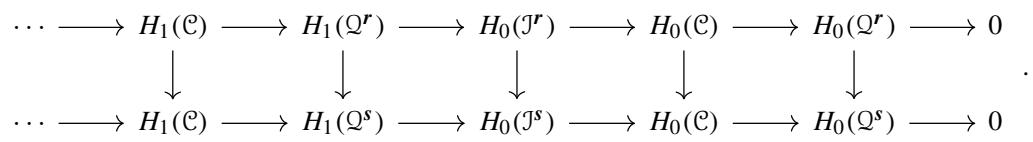


By an application of the Five lemma [23, 25], we obtain that the map $H_{1}\left(Q^{r}\right) \rightarrow$ $H_{1}\left(Q^{s}\right)$ must be a surjection. Then, the claim follows from the lower-acyclicity of $Q^{r}$.

Corollary 3.3 If $H_{0}\left(\mathcal{J}^{s} / \mathcal{J}^{r}\right)=0$ and $Q^{r}$ is lower-acyclic, then $Q^{s}$ is lower-acyclic.

As mentioned earlier, $H_{0}\left(\mathcal{J}^{s} / \mathcal{J}^{r}\right)$ is a simpler object to study, both computationally and analytically. Let us precisely state what we mean by "easier." Let $\mathcal{T}_{1}^{s}$ be the set of edges $\tau$ for which $s(\tau)<\boldsymbol{r}(\tau)$, and let $\mathcal{T}_{0}^{s}$ be the set of vertices of the edges $\tau \in \mathcal{T}_{1}^{s}$ in $\stackrel{\circ}{\mathcal{T}}_{0}$.

Lemma 3.4 The complex $\mathcal{J}^{s} / \mathcal{J}^{r}$ is supported only on $\mathcal{T}_{1}^{S}$ and $\mathcal{T}_{0}^{s}$, i.e., zero vector spaces are associated to all edges $\tau \notin \mathcal{T}_{1}^{s}$ and vertices $\gamma \notin \mathcal{T}_{0}^{s}$.

Proof The claim follows from the definition of the complexes $\mathcal{J}^{s}$ and $\mathcal{J}^{r}$. Indeed, if $\boldsymbol{s}(\tau)=\boldsymbol{r}(\tau)$, then $\mathfrak{I}_{\tau}^{s}=\mathfrak{I}_{\tau}^{r}$ and the cokernel of the inclusion map from $\mathcal{J}^{r}$ to $\mathcal{J}^{s}$ is zero on $\tau$; similarly for the vertices.

Remark 3.5 Corollary 3.3 provides sufficient conditions for ensuring loweracyclicity of $Q^{s}$ given that of $Q^{r}$. In particular, it should be noted that it does not place any assumptions on the homologies of the complex $\mathcal{C}$. Thus, our approach is compatible with studies which assume $H_{1}(\mathcal{C})$ and $H_{0}(\mathcal{C})$ both vanish (e.g., [13]) as well as studies where such assumptions are not made (e.g., [12]).

\section{Applications}

Let us now see how we can compute the dimension of splines on interesting meshes using Corollary 3.3. This result is quite general and is applicable in a large number of settings. We will narrow our focus down to the case where we reduce the smoothness across one or more interior edges $\tau$ from $\boldsymbol{r}(\tau)$ to $s(\tau)=-1$. This is motivated as follows. Let us say that we are working with splines on a polygonal partition $\mathcal{T}$ of a topological disk $\Omega$. Suppose we are able to reduce smoothness across all boundary edges of a number of polygonal faces of $\mathcal{T}$ to -1 while retaining lower-acyclicity (by an application of Corollary 3.3). Then we can carve out these polygons from the domain $\Omega$ to create a new mesh of more complex topology, and we can do so without losing the ability to exactly compute the dimension of splines on the new mesh. We now make this precise.

Definition 4.1 (Pruned mesh, spline space, and BSS complex) Given $\mathcal{T}, \boldsymbol{r}$ and $\boldsymbol{m}$, let $F \subset \mathcal{T}_{2}$ be the set of faces $\sigma$ such that, for any edge $\tau \subset \bar{\sigma}, \boldsymbol{r}(\tau)=-1$.

- A pruned mesh $\hat{\mathcal{T}}$ is obtained from $\mathcal{T}$ by deleting all faces in $F$.

- With $\hat{\boldsymbol{r}}$ and $\hat{\boldsymbol{m}}$ defined by restricting $\boldsymbol{r}$ and $\boldsymbol{m}$ to the edges and face of $\hat{\mathcal{T}}$, the spline space $\mathcal{R}_{\hat{\boldsymbol{m}}}^{\hat{r}}(\hat{\mathfrak{T}})$ will be called the pruned spline space. 
- $\quad$ The pruned BSS complex $\hat{Q}^{\hat{\boldsymbol{r}}}$ is defined on $\hat{\mathcal{T}}$ using $\hat{\boldsymbol{r}}$ and $\hat{\boldsymbol{m}}$. Equivalently, $\hat{Q}^{\hat{\boldsymbol{r}}}$ can be obtained from $Q^{r}$ by deleting all faces $\sigma \in F$ from the top vector space.

Note that, in general, the domains corresponding to a mesh $\mathcal{T}$ and its corresponding pruned mesh $\hat{\mathcal{T}}$ will be topologically different. The next three subsections show how the dimension of pruned spline spaces can be be computed for triangulations, polygonal meshes, and T-meshes. We will use the following result in all sections.

Theorem 4.2 Let $Q^{\boldsymbol{r}}$ be lower-acyclic for a given mesh $\mathcal{T}$, and let $\mathcal{R}_{\hat{\boldsymbol{m}}}^{\hat{\boldsymbol{r}}}(\hat{\mathcal{T}})$ and $\hat{Q}^{\hat{\boldsymbol{r}}}$ be the corresponding pruned spline space and BSS complex. Then,

$$
\operatorname{dim}\left(\mathcal{R}_{\hat{\boldsymbol{m}}}^{\hat{\boldsymbol{r}}}(\hat{\mathfrak{T}})\right)=\chi\left(\mathcal{Q}^{\boldsymbol{r}}\right)-\sum_{\sigma \in F} \operatorname{dim}\left(\mathcal{P}_{\sigma}\right) .
$$

Proof From Definition 2.3, and compared to the pruned spline space, the spline space $\mathcal{R}_{\boldsymbol{m}}^{\boldsymbol{r}}(\mathcal{T})$ does not impose any additional smoothness constraints along the edges of faces $\sigma \in F$. Then, and since Theorem 2.7 applies as $Q^{r}$ is lower-acyclic, we see that

$$
\chi\left(\mathcal{Q}^{r}\right)=\operatorname{dim}\left(\mathcal{R}_{\boldsymbol{m}}^{\boldsymbol{r}}(\mathcal{T})\right)=\operatorname{dim}\left(\mathcal{R}_{\hat{\boldsymbol{m}}}^{\hat{r}}(\hat{\mathcal{T}})\right)+\sum_{\sigma \in F} \operatorname{dim}\left(\mathcal{P}_{\sigma}\right)
$$

\subsection{Triangulations}

In this section, we show that Corollary 3.3 and Theorem 4.2 can be used to recover dimension formulas for splines on triangulations with holes. For instance, Theorem 4.9 shows that the complex $Q^{r}$ associated to the space of $C^{r}$ splines of degree $m \geq 3 r+2$ is always lower-acyclic. Similarly, Corollary 4.10 provides finer control over the dimension of splines than was previously known if there are enough slopes meeting at interior vertices. Note that the former result is not new-a dimension formula for splines on planar triangulations with holes is given in [20] for $m \geq 4 r+1$. This formula is extended to $m \geq 3 r+2$ in [21] where an extension of Schumaker's lower bound to rectilinear partitions with holes (and possibly non-triangular faces) is also made. Thus, the main purpose of Theorem 4.9 is to illustrate the utility of Corollary 3.3 by providing an alternate proof of the fact. Moreover, this section also sets the stage for our treatment of arbitrary rectilinear partitions in Section 4.2.

In the interest of a focused discussion, we consider meshes $\mathcal{T}$ which satisfy the following properties:

$$
\mathcal{P}_{\sigma}=\mathcal{P}_{m} \quad \forall \sigma \in \mathcal{T}_{2} \quad \text { and } \quad \boldsymbol{r}(\tau) \in\{r,-1\} \quad \forall \tau \in \stackrel{\circ}{\mathcal{T}}_{1},
$$

where $m \in \mathbb{Z}_{\geqslant 0}, r \in \mathbb{Z}_{\geqslant-1}$, and $\mathcal{P}_{m}$ is the space of polynomials of total degree at most $m$.

Lemma 4.3 Let $Q^{r}$ be lower-acyclic, and consider an interior edge $\tau$ with end-points $\gamma$ and $\gamma^{\prime}$ such that $\boldsymbol{r}(\tau)=r$. Let $\boldsymbol{s}$ be the smoothness distribution obtained from $\boldsymbol{r}$ by 
reducing the smoothness across $\tau$ to -1 , i.e.,

$$
\boldsymbol{s}\left(\tau^{\prime \prime}\right):= \begin{cases}\boldsymbol{r}\left(\tau^{\prime \prime}\right), & \tau^{\prime \prime} \neq \tau, \\ -1, & \tau^{\prime \prime}=\tau .\end{cases}
$$

If there exists an edge $\tau^{\prime}$ incident on $\gamma$ such that $\boldsymbol{r}\left(\tau^{\prime}\right)=-1$, then $Q^{s}$ is also loweracyclic.

\section{Proof From Lemma $2.4, \mathcal{J}^{s} / \mathcal{J}^{r}$ is}

- Supported on both $\tau$ and $\gamma^{\prime}$ if $\boldsymbol{r}\left(\tau^{\prime \prime}\right) \neq-1$ for any edge $\tau^{\prime \prime}$ incident on $\gamma^{\prime}$, and,

- Supported only on $\tau$ otherwise.

Then, for both these cases, it is easy to verify that $H_{0}\left(\mathcal{J}^{s} / \mathcal{J}^{r}\right)$ vanishes and the claim follows from Corollary 3.3.

Lemma 4.3 tells us that if an edge $\tau$ is incident on a vertex $\gamma$ such that $\boldsymbol{r}(\tau)=-1$, then for any other edge $\tau^{\prime}$ that is incident on $\gamma$, we can reduce the smoothness across $\tau^{\prime}$ to -1 preserving lower-acyclicity for all $m$. On the other hand, we now consider what happens when we reduce the smoothness across the entire boundary of a face $\sigma \in \mathcal{T}_{2}$ to -1 . That is, if $\boldsymbol{r}$ is a smoothness distribution on $\mathcal{T}_{1}$ and $\sigma$ is a face with bounding edges $\tau_{1}, \tau_{2}$, and $\tau_{3}$, we consider the smoothness distribution $s$ defined as

$$
s(\tau):= \begin{cases}\boldsymbol{r}(\tau), & \tau \neq \tau_{i}, i=1,2,3, \\ -1, & \text { otherwise. }\end{cases}
$$

We call $\boldsymbol{s}$ the smoothness distribution obtained from $\boldsymbol{r}$ by reducing smoothness across $\partial \sigma$ to -1 . Unlike Lemma 4.3, our result will now depend on $m$.

If we wish to reduce smoothness across the entire boundary of a triangular face to -1 while preserving lower-acyclicity using Corollary 3.3 , then we must describe $H_{0}\left(\mathcal{J}^{s} / \mathcal{J}^{r}\right)$. This homology is the cokernel of the only non-trivial map in the chain complex $\mathcal{J}^{s} / \mathcal{J}^{r}$; we call this map $\phi$. Suppose $\sigma$ is bounded by edges $\tau_{1}, \tau_{2}$, and $\tau_{3}$. Let $\gamma_{i}=\tau_{i} \cap \tau_{i+1}$ be interior vertices of $\mathcal{T}$, where the index $i$ is cyclic in $(1,2,3)$. Let $\ell_{i}$ be a linear form vanishing along $\tau_{i}$ for $i=1,2,3$. Then, $\phi$ is the map:

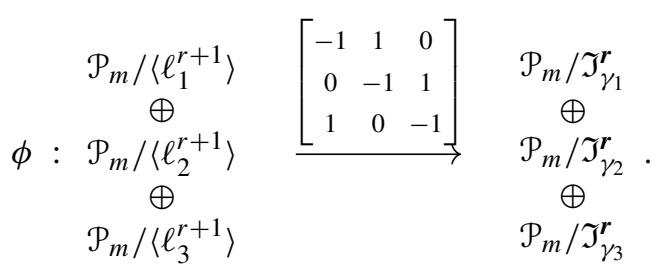

Here, $\left\langle\ell_{i}^{r+1}\right\rangle$ is the subspace of $\mathcal{P}_{m}$ containing all polynomial multiples of $\ell_{i}^{r+1}$, for $i=1,2,3$ (see also Remark 4.6).

Lemma 4.4 Let $Q^{r}$ be lower-acyclic and consider face $\sigma$, edges $\tau_{i}$, and vertices $\gamma_{i}$, $i=1,2,3$, as defined above. Assume that the following hold for all $i \in\{1,2,3\}$ :

$-\boldsymbol{r}\left(\tau_{i}\right)=r$,

- $\boldsymbol{r}(\tau) \neq-1$ for any other edge $\tau$ incident on $\gamma_{i}$, 
Let $\boldsymbol{s}$ be the smoothness distribution obtained from $\boldsymbol{r}$ by reducing the smoothness across $\partial \sigma$ to -1 . If the cokernel of the map $\phi$ from Equation (4.1) is trivial, then $Q^{s}$ is lower-acyclic.

Proof The claim is immediate from Corollary 3.3 and the fact that $H_{0}\left(\mathcal{J}^{s} / \mathcal{J}^{r}\right)$ is precisely the cokernel of the map $\phi$ from Equation (4.1).

Lemma 4.5 The cokernel of the map $\phi$ in Equation (4.1), and hence $H_{0}\left(\mathcal{J}^{s} / \mathcal{J}^{r}\right)$, is isomorphic to

$$
\mathcal{P}_{m} /\left(\mathfrak{I}_{\gamma_{1}}^{r}+\mathfrak{I}_{\gamma_{2}}^{\boldsymbol{r}}+\mathfrak{I}_{\gamma_{3}}^{\boldsymbol{r}}\right)
$$

Proof We apply the snake lemma to the left two columns of the following commutative diagram:

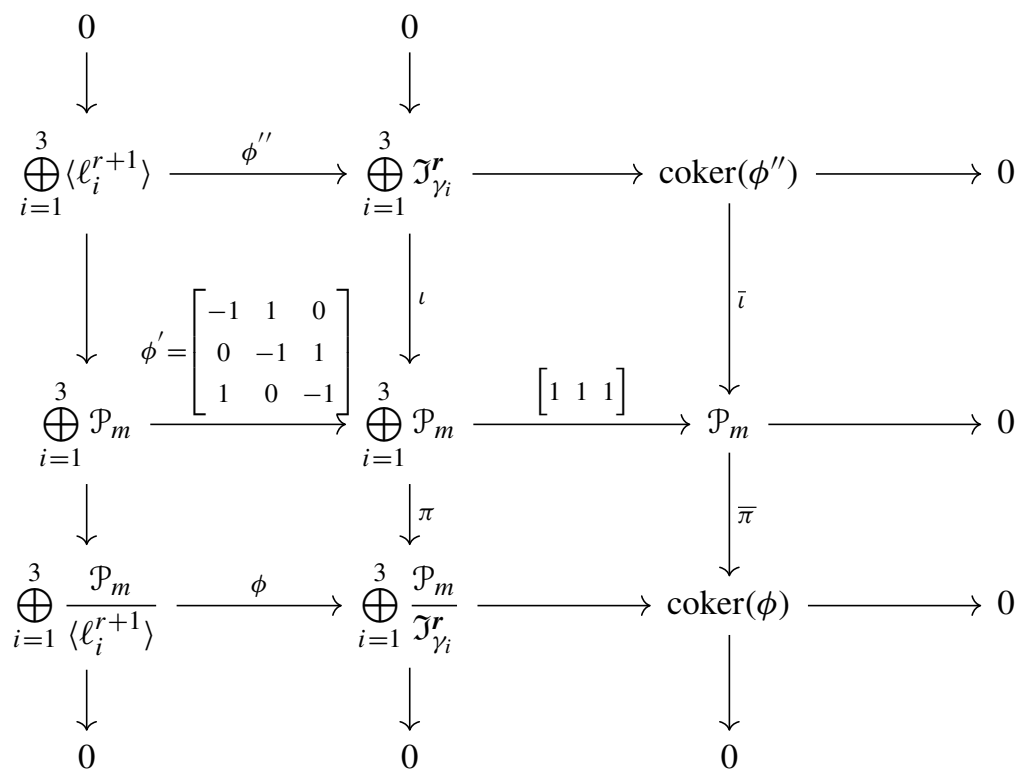

We only need the last portion of the snake lemma, namely the rightmost vertical column given by the sequence

$$
\operatorname{coker} \phi^{\prime \prime} \stackrel{\bar{\iota}}{\rightarrow} \operatorname{coker} \phi^{\prime} \stackrel{\bar{\pi}}{\rightarrow} \operatorname{coker}(\phi) \rightarrow 0,
$$

where $\bar{\imath}$ and $\bar{\pi}$ are the induced maps from $\iota$ and $\pi$. In other words, $\operatorname{coker}(\phi) \cong$ coker $(\bar{l})$. The above diagram explicitly identifies coker $\phi^{\prime}$ with $\mathcal{P}_{m}$. Since the diagram is commutative, $\bar{\imath} \cong\left[\begin{array}{lll}1 & 1 & 1\end{array}\right] \circ \iota$. The image of the latter inside $\mathcal{P}_{m}$ is clearly $\mathfrak{I}_{\gamma_{0}}^{r}+$ $\mathfrak{I}_{\gamma_{1}}^{r}+\mathfrak{I}_{\gamma_{2}}^{r}$. Thus

$$
\operatorname{coker}(\phi) \cong \operatorname{coker}(\bar{l}) \cong \mathcal{P}_{m} /\left(\mathfrak{I}_{\gamma_{0}}^{r}+\mathfrak{I}_{\gamma_{1}}^{r}+\mathfrak{I}_{\gamma_{2}}^{r}\right)
$$

as claimed. 
Remark 4.6 In the remainder of the manuscript, if $f_{1}, \ldots, f_{k}$ are polynomials in $\mathcal{P}_{m}$ we use the notation $\left\langle f_{i}\right\rangle$ to denote the subspace of $\mathcal{P}_{m}$ containing all polynomial multiples of $f_{i}$ and $\left\langle f_{1}, \ldots, f_{k}\right\rangle$ to denote $\sum_{i=1}^{k}\left\langle f_{i}\right\rangle$.

By a change of coordinates, we may assume that $\ell_{1}=x, \ell_{2}=y$, and $\ell_{3}=z$, where $z=(x+y+1)$. Then

$$
\begin{aligned}
& \mathfrak{I}_{\gamma_{1}}^{r}=\left\langle x^{r+1}, y^{r+1}, L_{1}^{r+1}, L_{2}^{r+1}, \cdots, L_{t_{1}-2}^{r+1}\right\rangle \\
& \mathfrak{I}_{\gamma_{2}}^{r}=\left\langle y^{r+1}, z^{r+1}, M_{1}^{r+1}, M_{2}^{r+1}, \cdots, M_{t_{2}-2}^{r+1}\right\rangle \\
& \mathfrak{I}_{\gamma_{3}}^{r}=\left\langle x^{r+1}, z^{r+1}, N_{1}^{r+1}, N_{2}^{r+1}, \cdots, N_{t_{3}-2}^{r+1}\right\rangle,
\end{aligned}
$$

where $L_{i}, M_{i}$, and $N_{i}$ are linear forms in $x$ and $y, y$ and $z$, and $x$ and $z$, respectively (regarding $z$ as a variable), and the integer $t_{i}$ is the maximum number of the powers of linear forms in $\left\{\ell_{\tau}^{r+1}: \gamma_{i} \in \tau\right\}$ which are linearly independent. It is known that $t_{i}$ is the minimum of $r+2$ and the number of slopes incident upon $\gamma_{i}$. A standard basis for $\mathcal{P}_{m}$ is provided by the monomials $x^{i} y^{j}, i+j \leq m$. An alternative basis for $\mathcal{P}_{m}$ which is more convenient for our arguments is the polynomials $x^{i} y^{j} z^{k}=x^{i} y^{j}(x+y+1)^{k}$, where $i+j+k=d$. Lemma 4.5 guarantees that $H_{0}\left(\mathcal{J}^{s} / \mathcal{J}^{r}\right)$ will vanish if every polynomial of this form is in the sum $\mathfrak{I}_{\gamma_{1}}^{r}+\mathfrak{I}_{\gamma_{2}}^{r}+\mathfrak{I}_{\gamma_{3}}^{r}$. We can obtain good estimates for this from the integers:

$$
\begin{aligned}
& \Omega_{1}:=\min \left\{d: x^{i} y^{j} \in \mathfrak{I}_{\gamma_{1}}^{r} \text { for all } i+j \geq d\right\}, \\
& \Omega_{2}:=\min \left\{d: y^{i} z^{j} \in \mathfrak{I}_{\gamma_{2}}^{r} \text { for all } i+j \geq d\right\}, \\
& \Omega_{3}:=\min \left\{d: x^{i} z^{j} \in \mathfrak{I}_{\gamma_{3}}^{r} \text { for all } i+j \geq d\right\} .
\end{aligned}
$$

We can in fact obtain these exactly using [9].

Lemma 4.7 (Schenck and Stillman [9, Corollary 3.4]) Let the vertices $\gamma_{1}, \gamma_{2}, \gamma_{3}$ be as above. Define $t_{i}$ and $\Omega_{i}$ for $i=1,2,3$ as above. Then, for $i=1,2,3$,

$$
\Omega_{i}=r+\left\lceil\frac{r+1}{t_{i}-1}\right\rceil .
$$

We are now in a position to state our main result on triangulations.

Theorem 4.8 Let $Q^{r}$ be lower-acyclic and consider a face $\sigma$, edges $\tau_{i}$ and vertices $\gamma_{i}, i=1,2,3$, as defined above. Assume that the following hold for all $i \in\{1,2,3\}$,

$-\boldsymbol{r}\left(\tau_{i}\right)=r$,

- $\boldsymbol{r}(\tau) \neq-1$ for any other edge $\tau$ incident on $\gamma_{i}$,

Suppose $\gamma_{i}$ has $n_{i}$ distinct slopes, and define $t_{i}=\min \left\{r+1, n_{i}\right\}$ and $\Omega_{i}=r+\left\lceil\frac{r+1}{t_{i}-1}\right\rceil$ for $i=1,2,3$. Let $\boldsymbol{s}$ be the smoothness distribution obtained from $\boldsymbol{r}$ by reducing smoothness across $\partial \sigma$ to -1 . Then, if $m>\frac{\Omega_{1}+\Omega_{2}+\Omega_{3}-3}{2}$, the chain complex $Q^{s}$ for the pruned mesh is lower-acyclic.

Proof By Lemma 4.4, it suffices to show that the cokernel of the map $\phi$ in Equation 4.1 is trivial. By Lemma 4.5 it suffices to show that $\mathfrak{I}_{\gamma_{1}}^{r}+\mathfrak{I}_{\gamma_{1}}^{r}+\mathfrak{I}_{\gamma_{2}}^{r}=\mathcal{P}_{m}$ for 
$m>\frac{\Omega_{1}+\Omega_{2}+\Omega_{3}-3}{2}$. Suppose that $x^{i} y^{j} z^{k} \notin \mathfrak{I}_{\gamma_{1}}^{r}+\mathfrak{I}_{\gamma_{1}}^{r}+\mathfrak{I}_{\gamma_{2}}^{r}$. Then, by Lemma 4.7, we must have

$$
\begin{aligned}
& i+j \leq \Omega_{1}-1 \\
& j+k \leq \Omega_{2}-1 \\
& i+k \leq \Omega_{3}-1 .
\end{aligned}
$$

Summing these leads to $2(i+j+k) \leq \Omega_{1}+\Omega_{2}+\Omega_{3}-3$, or $m=(i+j+k) \leq$ $\frac{\Omega_{1}+\Omega_{2}+\Omega_{3}-3}{2}$. Thus, $\mathfrak{I}_{\gamma_{1}}^{r}+\mathfrak{I}_{\gamma_{1}}^{r}+\mathfrak{I}_{\gamma_{2}}^{r}=\mathcal{P}_{m}$ for $m>\frac{\Omega_{1}+\Omega_{2}+\Omega_{3}-3}{2}$. By Lemmas 4.4 and $4.5, Q^{s}$ is lower acyclic for $m>\frac{\Omega_{1}+\Omega_{2}+\Omega_{3}-3}{2}$.

Theorem 4.9 Suppose $\mathcal{T}$ is any triangulation. Then $Q^{r}$ is lower-acyclic for $m \geq 3 r+$ 2. In particular, the formula for $\operatorname{dim}\left(\mathcal{R}^{r}\right)$ in [24, Equation 7.1] holds for $m \geq 3 r+2$.

Proof An arbitrary planar triangulation $\mathcal{T}$ has some number of holes, along with a minimal number of triangles needed to "fill in" those holes with a triangulation. We induct on this minimal number of triangles needed to "fill in" the holes. If no such triangles are needed, then $\mathcal{T}$ triangulates a simply connected region and the main result of [26] (see also [27] where a similar result is shown for superspline spaces) implies that the corresponding chain complex $Q^{r}$ is lower-acyclic for $m \geq 3 r+2$. Now suppose $\mathcal{T}$ triangulates an arbitrary non-simply connected region with corresponding chain complex $Q^{s}$. Pick one of the holes in $\mathcal{T}$ and form $\mathcal{T}^{\prime}$ by adding in a triangular face $\sigma$ so that:

1. $\sigma$ begins a filling of the holes of $\mathcal{T}$ in a minimal fashion

2. $\bar{\sigma} \cap \mathcal{T}$ is connected

By induction, the chain complex $Q^{r}$ corresponding to $\mathcal{T}^{\prime}$ is lower-acyclic for $m \geq$ $3 r+2$. If $\bar{\sigma} \cap \mathcal{T}$ is not the entire boundary of $\sigma$, then applying Lemma 4.3 at most twice yields that $Q^{s}$ is lower-acyclic for $m \geq 3 r+2$. If $\bar{\sigma} \cap \mathcal{T}$ is the entire boundary of $\sigma$, then applying Theorem 4.8 yields that $Q^{s}$ is lower-acyclic for $m \geq 3 r+2$ as long as $\left(\Omega_{1}+\Omega_{2}+\Omega_{3}-3\right) / 2<3 r+2$. The integers $\Omega_{i}$ are largest when only two slopes meet at $\gamma_{i}$. In this case, $\Omega_{i}=2 r+1$. Hence

$$
\left(\Omega_{1}+\Omega_{2}+\Omega_{3}-3\right) / 2 \leq(6 r) / 2=3 r<3 r+2,
$$

completing the induction.

The last statement in Theorem 4.9 now follows from Theorem 2.7 and Theorem 4.2, since the Euler characteristic of $Q^{r}$ (subtracting off the dimensions $\operatorname{dim}\left(\mathcal{P}_{m}\right)$ for each triangle used to fill in the holes) is precisely the dimension of the spline space. The exact formulas can also be derived from the chain complex; see [9, 28]. We do not discuss this here.

Lemma 4.5 can be used to give better estimates in non-generic situations for combinations of low degree and high smoothness, as we illustrate in the following corollary. 
Corollary 4.10 Let $r, m$, and $\mathcal{T}$ be such that $Q^{r}$ is lower-acyclic. If every interior vertex of $\mathcal{T}$ has at least $r+2$ distinct slopes incident upon it and $m>\frac{3 r}{2}$ then the smoothness across the boundary of any face $\sigma \in \mathcal{T}_{2}$ can be reduced to -1 while preserving lower-acyclicity. In particular, the following can be said about specific choices of $r$ and $m$.

(A) $\quad m \geqslant 2$ and $r=1$ : If $\mathcal{T}$ is such that any interior vertex has edges with at least 3 distinct slopes incident upon it, then the smoothness across the boundary of any face $\sigma \in \mathcal{T}_{2}$ can be reduced to -1 while preserving lower-acyclicity.

(B) $\quad m \geqslant 4$ and $r=2$ : If $\mathcal{T}$ is such that any interior vertex has edges with at least 4 distinct slopes incident upon it, then the smoothness across the boundary of any face $\sigma \in \mathcal{T}_{2}$ can be reduced to -1 while preserving lower-acyclicity.

In all such cases, the dimension of the corresponding pruned spline space can be computed by a direct application of Theorem 4.2.

Example 4.11 ( $C^{1}$ quadratic splines on a domain with holes) Consider the meshes shown in Fig. 3. We are interested in the space of $C^{1}$ quadratic splines on mesh in Fig. 3(a). Then, we can interpret this space as the pruned version of the space of $C^{1}$ quadratics on the triangulation in Fig. 3(b) after we have reduced the smoothness across the dashed edges in Fig. 3(c) to -1 . Therefore, we start by looking at the mesh in Fig. 3(b).

First, for the mesh in Fig. 3(b), choose all $\mathcal{P}_{\sigma}=\mathcal{P}_{2}$ for all faces $\sigma$, where $\mathcal{P}_{2}$ is the space of polynomials of total degree at most 2 , and $\boldsymbol{r}(\tau)=1$ for all interior edges $\tau$. It can be checked, using the formulas in [29], for instance, that $Q^{r}$ is lower-acyclic and the dimension of the corresponding space is 27 .

Then, using Lemmas 4.3 and 4.4, we can reduce the smoothness across all dashed edges in Fig. 3(c) to -1 while preserving lower-acyclicity (c.f. Corollary 4.10(A)).

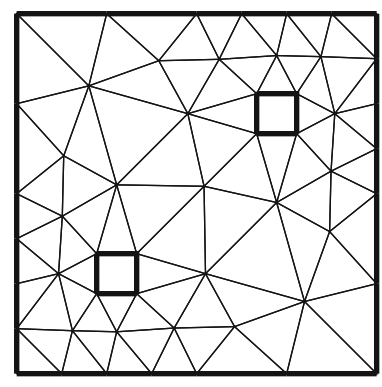

(a)

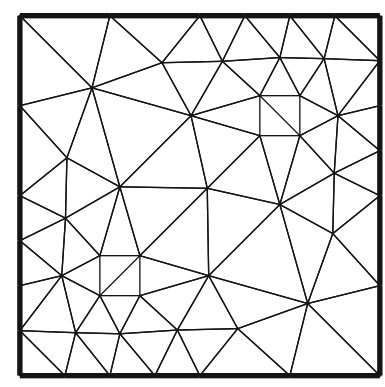

(b)

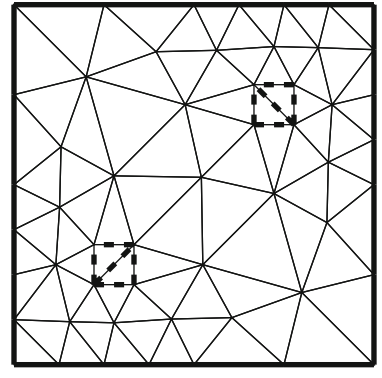

(c)

Fig. 3 A non-simply connected domain and its triangulation are shown in a. Starting from the triangulation in $\mathbf{b}$, we can interpret the triangulation in $\mathbf{a}$ as a pruned triangulation once the smoothness across the dashed edges in $\mathbf{c}$ have been reduced to -1 . In $\mathbf{c}$, reducing the smoothness to -1 decouples the faces inside the dashed region from those outside the dashed region. Then, the required spline space dimension can be computed from the one on $\mathbf{c}$ using Theorem 4.2, i.e., by subtracting $\operatorname{dim}\left(\mathcal{P}_{\sigma}\right)$ for each $\sigma$ contained inside the dashed region. Note that all domain boundaries have been displayed in bold 
The dimension of the resulting space is 53. As a result, the dimension of the pruned space in Fig. 3(a) can be exactly computed using Theorem 4.2 to be 29 .

Note that, for the mesh in Fig. 3(a), $H_{1}(\mathcal{C})$ is not 0 . Therefore, it is not directly covered by the approach presented in [29] for dimension counting. The result of the computation of course coincides with Billera [7, Theorem 5.8] since we looked at the special choice of $r=1$. Nonetheless, our results can also be applied for different choices of $\boldsymbol{r}$; c.f. Corollary 4.10(B).

Remark 4.12 At first glance, it may seem strange that the dimension of splines in Fig. $3 b$ is smaller than the dimension of splines in Fig. 3(a). However, this makes sense because, while removing some faces from the mesh, we also removed the smoothness constraints across the boundaries of those faces. The net effect of such operations may very well lead to an increase in the dimension, as is the case here. The same observation will also hold later when we look at T-meshes in Example 4.28.

\subsection{Polygonal meshes}

We now turn to planar meshes with convex polygonal faces, or rectilinear meshes. We again show that Theorem 4.2 and Corollary 3.3 can be used to recover dimension formulas for splines on rectilinear meshes with holes (and $m$ large enough-this will be specified later) from dimensions formulas for splines on rectilinear meshes without holes. A dimension formula for splines on polygonal meshes without holes (and $m \gg 0$ ) is derived in [10]; in [11] it is shown that this formula holds for $m \geq(2 F+1)(r+1)-2$, where $F$ is the largest number of edges surrounding a single face of $\mathcal{T}$. In practice, the dimension formula in [10] typically holds for much smaller values of $m$, as we will see in Example 4.16. For most vertex positions, the dimension formula of [10] agrees with Schumaker's lower bound from [5] when $m \gg 0$.

Let $\mathcal{T}$ be an arbitrary rectilinear mesh (allowing polygonal faces) with the same setup as in Section 4.1. That is,

$$
\mathcal{P}_{\sigma}=\mathcal{P}_{m} \quad \forall \sigma \in \mathcal{T}_{2} \quad \text { and } \quad \boldsymbol{r}(\tau) \in\{r,-1\} \quad \forall \tau \in \stackrel{\circ}{\mathcal{T}}_{1},
$$

where $m \in \mathbb{Z}_{\geqslant 0}, r \in \mathbb{Z}_{\geqslant-1}$, and $\mathcal{P}_{m}$ is the space of polynomials of total degree at most $m$. We will assume that the polygonal faces are convex although this condition could be dropped for particular examples.

We consider reducing smoothness to -1 across the boundary of a face $\sigma \in \mathcal{T}$. Suppose $\sigma$ is bounded by edges $\tau_{1}, \tau_{2}, \ldots, \tau_{k}$. Let $\gamma_{i}=\tau_{i} \cap \tau_{i+1}$ be interior vertices of $\mathcal{T}$, where the index $i$ is cyclic in $(1,2, \ldots, k)$. Let $\ell_{i}$ be a linear form vanishing along $\tau_{i}$ for $i=1,2, \ldots, k$. Let $s$ be the smoothness distribution where:

$$
s(\tau):= \begin{cases}\boldsymbol{r}(\tau), & \tau \neq \tau_{i}, i=1, \ldots, k \\ -1, & \text { otherwise. }\end{cases}
$$


We again call $\boldsymbol{s}$ the smoothness distribution obtained from $\boldsymbol{r}$ by reducing smoothness across $\partial \sigma$ to -1 . Then, the only non-trivial map $\phi$ in $H_{0}\left(\mathcal{J}^{\mathcal{S}} / \mathcal{J}^{r}\right)$ is given by

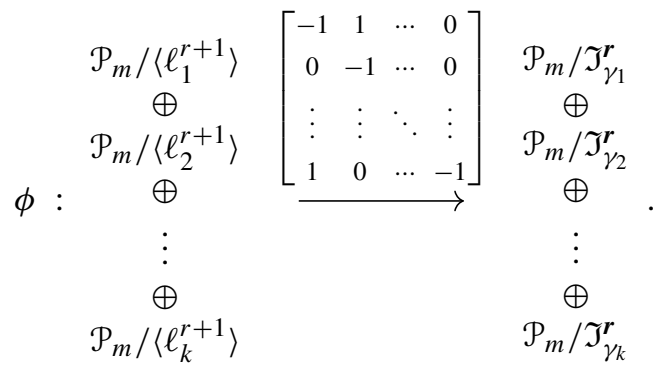

Lemma 4.13 The cokernel of $\phi$, and hence $H_{0}\left(\mathcal{J}^{s} / \mathcal{J}^{\boldsymbol{r}}\right)$, is isomorphic to $\mathcal{P}_{m} /\left(\mathfrak{I}_{\gamma_{1}}^{\boldsymbol{r}}+\right.$ $\left.\cdots+\mathfrak{I}_{\gamma_{k}}^{r}\right)$.

Proof The proof is the same as Lemma 4.5.

If the face $\sigma$ is not triangular, then it is likely that $\left(\mathfrak{I}_{\gamma_{1}}^{r}+\cdots+\mathfrak{I}_{\gamma_{k}}^{r}\right)=\mathcal{P}_{m}$ for quite small $m$ relative to $r$ and it is possible to obtain quite accurate estimates for the smallest such $m$. However, this equality typically holds in degree far lower than dimension formulas are actually known (see [11]), so we focus on giving some coarse estimates that are easy to derive.

Proposition 4.14 Let $\mathcal{T}$ be a planar polygonal mesh and $\sigma$ a face of $\mathcal{T}$ with bounding edges $\tau_{1}, \ldots, \tau_{k}$ and vertices $\gamma_{i}=\tau_{i} \cap \tau_{i+1}$, with $i$ taken cyclically from $(1, \ldots, k)$. Let $\boldsymbol{r}$ be a fixed smoothness distribution and let $\boldsymbol{s}$ be the smoothness distribution obtained from $\boldsymbol{r}$ by reducing smoothness across $\partial \sigma$ to -1 . As above, let $\Omega_{i}=r+$ $\left\lceil\frac{r+1}{t_{i}-1}\right\rceil$, where $t_{i}=\min \left\{r+1, n_{i}\right\}$ and $n_{i}$ is the number of distinct slopes incident at $\gamma_{i}$. Then $H_{0}\left(\mathcal{J}^{s} / \mathcal{J}^{r}\right)$ vanishes for

- $m>3 r$ or

- $m>\Omega_{i}+\Omega_{i+1}-2$ for any $i=1, \ldots, k$.

In particular, if either of these conditions holds and $Q^{r}$ is lower-acyclic then so is $Q^{s}$.

Proof By Lemma 4.13, it suffices to show that $\left(\mathfrak{I}_{\gamma_{1}}^{r}+\cdots+\mathfrak{I}_{\gamma_{k}}^{r}\right)=\mathcal{P}_{m}$ for the two cases above.

Without loss of generality, we can change coordinates so that $\mathfrak{I}_{\tau_{1}}^{r}=\left\langle x^{r+1}\right\rangle, \mathfrak{I}_{\tau_{2}}^{r}=$ $\left\langle y^{r+1}\right\rangle$, and $\mathfrak{I}_{\tau_{i}}^{r}=\left\langle z^{r+1}\right\rangle$ for some $2<i<k$, where $z=(x+y+1)$. Then, it is clear that $\left\langle x^{r+1}, y^{r+1}, z^{r+1}\right\rangle \subset\left(\mathfrak{I}_{\gamma_{1}}^{r}+\cdots+\mathfrak{I}_{\gamma_{k}}^{r}\right)$. We again choose to use the basis $x^{i} y^{j} z^{k}, i+j+k=m$, for $\mathcal{P}_{m}$. If $x^{i} y^{j} z^{k} \notin\left\langle x^{r+1}, y^{r+1}, z^{r+1}\right\rangle$, then we must have $i \leq r, j \leq r$ and $k \leq r$; thus $i+j+k \leq 3 r$. It follows that $\left\langle x^{r+1}, y^{r+1}, z^{r+1}\right\rangle=\mathcal{P}_{m}$, hence also $\left(\mathfrak{I}_{\gamma_{1}}^{r}+\cdots+\mathfrak{I}_{\gamma_{k}}^{r}\right)=\mathcal{P}_{m}$. 
Now suppose that $m>\Omega_{i}+\Omega_{i+1}-2$ for some $i=1, \ldots, k$. Again, changing coordinates, we may assume that:

$$
\begin{aligned}
\mathfrak{I}_{\gamma_{i}}^{r} & =\left\langle x^{r+1}, y^{r+1}, L_{1}^{r+1}, L_{2}^{r+1}, \cdots, L_{t_{1}-2}^{r+1}\right\rangle \\
\mathfrak{I}_{\gamma_{i+1}}^{r} & =\left\langle y^{r+1}, z^{r+1}, M_{1}^{r+1}, M_{2}^{r+1}, \cdots, M_{t_{2}-2}^{r+1}\right\rangle
\end{aligned}
$$

where $L_{i}$ and $M_{i}$ are linear forms in $x$ and $y$, and $y$ and $z$, respectively (regarding $z$ as a variable, where we are again letting $z=x+y-1)$. Recall that $x^{i} y^{j} z^{k}$, $i+j+k=m$, form a basis for $\mathcal{P}_{m}$. Suppose $x^{i} y^{j} z^{k} \notin \mathfrak{I}_{\gamma_{i}}^{r}+\mathfrak{I}_{\gamma_{i+1}}^{r}$. Then $i+j \leq \Omega_{i}-1$ and $j+k \leq \Omega_{i+1}-1$. Hence, $i+j+k \leq i+2 j+k \leq \Omega_{i}+\Omega_{i+1}-2$, contrary to assumption. Hence $\mathfrak{I}_{\gamma_{i}}^{r}+\mathfrak{I}_{\gamma_{i+1}}^{r}=\mathcal{P}_{m}$, and thus $\left(\mathfrak{I}_{\gamma_{1}}^{r}+\cdots+\mathfrak{I}_{\gamma_{k}}^{r}\right)=\mathcal{P}_{m}$ as well.

Remark 4.15 Suppose $\mathcal{T}$ is a polygonal mesh with smoothness distribution $\boldsymbol{r}$. Let $\boldsymbol{s}$ be the smoothness distribution which is equal to $\boldsymbol{r}$ on every edge other than $\tau$, and satisfies $s(\tau)=-1$. Suppose $\tau$ joins vertices $\gamma_{1}$ and $\gamma_{2}$. Let $\Omega_{i}=r+\left\lceil\frac{r+1}{t_{i}-1}\right\rceil$, where $t_{i}=\min \left\{r+1, n_{i}\right\}$ and $n_{i}$ is the number of distinct slopes incident at $\gamma_{i}(i=1,2)$. If $m>\Omega_{1}+\Omega_{2}-2$, the proof of Proposition 4.14 shows that if $Q^{r}$ is lower-acyclic then so is $Q^{s}$. This observation can be used to remove all smoothness requirements along arbitrary edges, as long as $m$ is large enough.

Example 4.16 ( $C^{1}$ splines on a polygonal mesh with holes) Let $\mathcal{T}$ be the mesh depicted in Fig. 4(b). For simplicity, we assume the coordinates of the vertices in this figure are chosen generically (thus $Q^{r}$ is lower-acyclic for large $m$ by [10]). In this case, [10] implies that $\operatorname{dim} \mathcal{R}^{1}=\left(\begin{array}{c}m+2 \\ 2\end{array}\right)-20\left(\begin{array}{c}m \\ 2\end{array}\right)+32\left(\begin{array}{c}m-1 \\ 2\end{array}\right)$ for $m \gg 0$; here $\left(\begin{array}{l}n \\ 2\end{array}\right)=\frac{n(n-1)}{2}$ is the binomial coefficient. The main result of [11] implies that $\operatorname{dim} \mathcal{R}^{1}=\left(\begin{array}{c}m+2 \\ 2\end{array}\right)-20\left(\begin{array}{c}m \\ 2\end{array}\right)+32\left(\begin{array}{c}m-1 \\ 2\end{array}\right)$ for $m \geq(2 \cdot 8-1) \cdot 2-2=28$. This bound from [11] takes into account worst-case scenarios and thus is typically much larger than it needs to be. Hence, for this particular example, we do a direct computation.

We can compute the dimension of splines on this mesh using the AlgebraicSplines package for Macaulay2 [30]. Doing this for a generic choice of coordinates for the vertices in Fig. 4, we find that $Q^{r}$ is lower-acyclic for $m \geq 7$; in other words, $\operatorname{dim} \mathcal{R}^{1}=\left(\begin{array}{c}m+2 \\ 2\end{array}\right)-20\left(\begin{array}{c}m \\ 2\end{array}\right)+32\left(\begin{array}{c}m-1 \\ 2\end{array}\right)$ for $m \geq 7$. Taking this improved bound for the lower-acyclicity of $Q^{r}$, we now use Proposition 4.14. For each of the vertices $\gamma_{1}, \ldots, \gamma_{8}$ on the boundary of the central octagon, $\Omega_{i}=2$. Thus, the lower bound of $3 r=3$ is better than the lower bound of $\Omega_{i}+\Omega_{i+1}-2=4$. Either of these is less than 7; hence, Proposition 4.14 indicates that the pruned spline space over the mesh depicted in Fig. 4(a) will satisfy the dimension formula $-4\left(\begin{array}{c}m \\ 2\end{array}\right)+16\left(\begin{array}{c}m-1 \\ 2\end{array}\right)$ for $m \geq 7$. (In fact, if we again use the AlgebraicSplines package in Macaulay2 to compute the dimension of the pruned spline space, we see that the dimension of the pruned spline space satisfies the formula $-4\left(\begin{array}{c}m \\ 2\end{array}\right)+16\left(\begin{array}{c}m-1 \\ 2\end{array}\right)$ for $m \geq 4$.)

\subsection{T-Meshes}

Let us now present examples of applications to splines on T-meshes $\mathcal{T}$. In particular, we will show how Corollary 3.3 can be combined with previously published results 


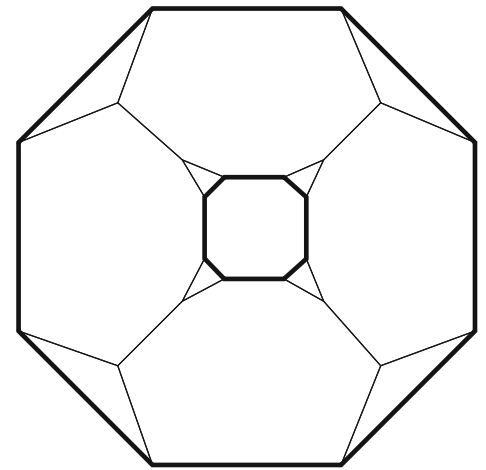

(a)

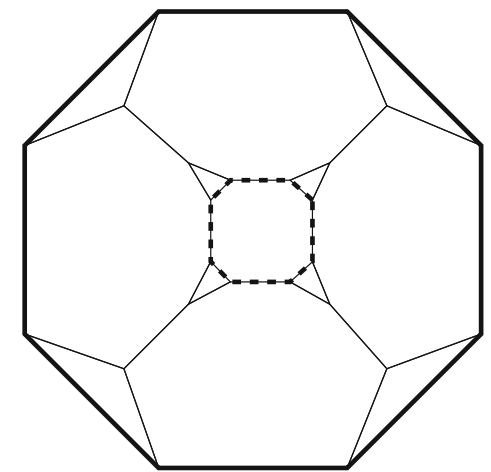

(b)

Fig. 4 A non-simply connected polygonal mesh is shown in a. We can interpret the mesh in a as a pruned mesh once the smoothness across the dashed edges in $\mathbf{b}$ have been reduced to -1 . In $\mathbf{b}$, reducing the smoothness to -1 decouples the faces inside the dashed region from those outside the dashed region. Then, the required spline space dimension can be computed from the one on a using Theorem 4.2, i.e., by subtracting the term $\operatorname{dim}\left(\mathcal{P}_{\sigma}\right)$ for the polygon enclosed by the dashed edges. All domain boundaries have been displayed in bold

from $[13,14,18]$ to compute the dimension of bi-degree splines in a very general setting by reducing the smoothness across one or more edges to -1 . Thereafter, Theorem 4.2 will allow us to compute the dimension of the corresponding pruned spline spaces on T-meshes of arbitrary topologies. In particular, in the simplified setting of maximally smooth, uniform degree splines on T-meshes with holes, Example 4.26 demonstrates the generality of our approach compared to the one of [16].

T-Meshes have a simpler structure and as a result we can consider a more general setting than the one we discussed in the previous sub-sections. More precisely, for $\sigma \in \mathcal{T}_{2}$, we will allow $\boldsymbol{m}(\sigma)=\mathcal{P}_{\sigma}$ to be the vector space of polynomials of bi-degree at most $\left(m_{\sigma}, m_{\sigma}\right)$ for some $m_{\sigma} \in \mathbb{Z}_{\geqslant 0}$, i.e.,

$$
\boldsymbol{m}(\sigma)=\mathcal{P}_{\sigma}=\mathcal{P}_{m_{\sigma} m_{\sigma}} .
$$

Of course, we will assume that the assumption placed on $\boldsymbol{m}$ in Eq. 2.1, Section 2, is still satisfied. Then, following Eq. 2.2, we have $\mathcal{P}_{\tau}=\mathcal{P}_{m_{\tau} m_{\tau}}$ and $\mathcal{P}_{\gamma}=\mathcal{P}_{m_{\gamma} m_{\gamma}}$, where we define:

$$
m_{\tau}:=\max _{\bar{\sigma} \supset \tau} m_{\sigma}, \quad m_{\gamma}:=\max _{\bar{\sigma} \ni \gamma} m_{\sigma} .
$$

We start by defining the segments of the T-mesh as connected unions of horizontal or vertical edges that have the same associated $m_{\tau}$.

Definition 4.17 (Segments of the T-mesh) Let $\rho \subseteq \stackrel{\circ}{\mathcal{T}}_{1}$ be a finite set of horizontal (resp. vertical) edges $\tau \in \stackrel{\mathfrak{T}}{1}_{1}$ :

- $\quad L_{\rho}:=\cup_{\tau \in \rho} \tau$ is non-empty and connected,

- $m_{\tau}=m_{\tau^{\prime}}=: m_{\rho}$ for any $\tau, \tau^{\prime} \in \rho$.

Then, $\rho$ will be called a horizontal (resp. vertical) segment of the mesh. 
Lemma 4.23, which will be presented shortly, identifies sufficient conditions allowing the smoothness across a segment of the T-mesh to be reduced while preserving lower-acyclicity. The next three results discuss the dimensions of spaces of univariate polynomials, and Example 4.21 presents an application of these results; the proof of Lemma 4.23 will use these results.

In the following, $\overline{\mathcal{P}}_{m}$ is used to denote the vector space of univariate polynomials in variable $x$ of degree at most $m ; \overline{\mathcal{P}}_{m}:=0$ for $m<0$. Finally, for $I=\{1, \ldots, k\}$ and some $a_{i} \in \mathbb{R}$ and $d_{i} \in \mathbb{Z}_{\geqslant-1}, i \in I$, we define $M(I) \subseteq I$ to be the largest set such that:

- All $a_{i}, i \in M(I)$, are distinct;

- For each $i \in M(I), d_{i}=\min \left\{d_{j}: a_{i}=a_{j}, j \in I\right\}$.

Lemma 4.18 (Proposition 1.8, Mourrain [13]) For $i \in I=\{1, \ldots, k\}$, let $a_{i} \in \mathbb{R}$ and $d_{i} \in \mathbb{Z}_{\geqslant-1}$. Consider linear polynomials $\ell_{i}=x-a_{i}, i \in I$, and define the vector space $V$ as

$$
V:=\sum_{i \in I} \ell_{i}^{d_{i}} \overline{\mathcal{P}}_{m-d_{i}}
$$

Then, the dimension of $V$ is given by the following formula:

$$
\operatorname{dim}(V)=\min \left(m+1, \sum_{i \in M(I)}\left(m-d_{i}+1\right)_{+}\right) .
$$

Lemma 4.19 For $i \in I=\{1, \ldots, k\}$, let $a_{i} \in \mathbb{R}, d_{i} \in \mathbb{Z}_{\geqslant-1}$ and $e_{i} \in \mathbb{Z}_{\geqslant 0}$. Consider linear polynomials $\ell_{i}=x-a_{i}, i \in I$, and define the vector space $V$ as below:

$$
V:=\sum_{i \in I} \ell_{i}^{d_{i}} \overline{\mathcal{P}}_{m-d_{i}-e_{i}}
$$

Then, the dimension of $V$ is given by the following formula:

$$
\begin{aligned}
& \operatorname{dim}(V)=\sum_{j=0}^{d}\left[\min \left(m-e^{j+1}+1, \sum_{i \in M\left(I^{j}\right)}\left(m-e^{j+1}-d_{i}+1\right)_{+}\right)\right. \\
&\left.-\min \left(m-e^{j}+1, \sum_{i \in M\left(I^{j}\right)}\left(m-e^{j}-d_{i}+1\right)_{+}\right)\right],
\end{aligned}
$$

where we use the following definitions:

$$
\begin{aligned}
E & :=\left\{e_{1}, \ldots, e_{k}\right\}, d:=\# E-1, \\
e^{j} & := \begin{cases}m+1, & j=0, \\
\max E \backslash\left\{e^{0}, \ldots, e^{j-1}\right\}, & j=1, \ldots, d+1,\end{cases} \\
I^{j} & :=\left\{i \in I: e_{i}<e^{j}\right\}, j=0, \ldots, d+1 .
\end{aligned}
$$

Proof The proof follows from Lemma 4.18 and [12, Lemma 4.5], where an analogous claim was shown for bivariate polynomials. 
Corollary 4.20 For $i \in I=\{1, \ldots, k\}$, let $a_{i} \in \mathbb{R}, d_{i} \in \mathbb{Z}_{\geqslant-1}$ and $e_{i} \in \mathbb{Z}_{\geqslant 0}$. Consider linear polynomials $\ell_{i}=x-a_{i}, i \in I$, and define the vector space $V$ as below:

If $\operatorname{dim}(V)=m+1$, then $V=\overline{\mathcal{P}}_{m}$.

$$
V:=\sum_{i \in I} \ell_{i}^{d_{i}} \overline{\mathcal{P}}_{m-d_{i}-e_{i}} .
$$

Example 4.21 As an illustration of Lemma 4.19, let us consider the vector space $V$ defined by choosing:

$$
\begin{aligned}
& m=3, \quad I=\{1,2,3,4\}, \quad\left(d_{1}, d_{2}, d_{3}, d_{4}\right)=(3,2,3,3), \\
& \left(e_{1}, e_{2}, e_{3}, e_{4}\right)=(0,1,0,0), \quad\left(a_{1}, a_{2}, a_{3}, a_{4}\right)=(-1,0,0,1) .
\end{aligned}
$$

That is, we choose $V$ as the following vector space, where $\ell_{i}=x-a_{i}$,

$$
V=\ell_{1}^{3} \overline{\mathcal{P}}_{0}+\ell_{2}^{2} \overline{\mathcal{P}}_{0}+\ell_{3}^{3} \overline{\mathcal{P}}_{0}+\ell_{4}^{3} \overline{\mathcal{P}}_{0}
$$

Then, following the definitions in Lemma 4.19, we have:

$$
\begin{aligned}
& E=\{0,1\}, \quad d=1, \quad\left(e^{0}, e^{1}, e^{2}\right)=(4,1,0), \\
& I=\{1,2,3,4\}, \quad I^{0}=I, \quad I^{1}=\{1,3,4\}, \quad I^{2}=\emptyset .
\end{aligned}
$$

Therefore, we see that:

$$
M\left(I^{0}\right)=\{1,2,4\}, \quad M\left(I^{1}\right)=\{1,3,4\},
$$

and the dimension of $V$ follows as:

$$
\begin{aligned}
\operatorname{dim}(V) & =\min \left(3-1+1, \sum_{i \in M\left(I^{0}\right)}\left(3-1-d_{i}+1\right)_{+}\right) \\
& -\min \left(3-4+1, \sum_{i \in M\left(I^{0}\right)}\left(3-4-d_{i}+1\right)_{+}\right) \\
& +\min \left(3-0+1, \sum_{i \in M\left(I^{1}\right)}\left(3-0-d_{i}+1\right)_{+}\right) \\
& -\min \left(3-1+1, \sum_{i \in M\left(I^{1}\right)}\left(3-1-d_{i}+1\right)_{+}\right) \\
& =(1-0)+(3-0)=4=\operatorname{dim}\left(\overline{\mathcal{P}}_{3}\right),
\end{aligned}
$$

so that $V=\overline{\mathcal{P}}_{3}$.

Definition 4.22 (Weight of a segment) Given a segment $\rho$, define the set $T$ as

$$
T=\left\{\bar{\tau} \in \mathcal{T}_{1}: \bar{\tau} \text { intersects } L_{\rho} \text { trasversally }\right\} .
$$

Let $a_{\bar{\tau}}$ be the horizontal (resp. vertical) coordinate for the vertical (resp. horizontal) edge $\bar{\tau}$. Then, the weight of $\rho, \omega^{r}(\rho)$, is defined as:

$$
\omega^{r}(\rho):=\operatorname{dim}\left(\sum_{\bar{\tau} \in T}\left(x-a_{\bar{\tau}}\right)^{r(\bar{\tau})+1} \overline{\mathcal{P}}_{m_{\rho}-\max \left(0, m_{\rho}-m_{\bar{\tau}}\right)-\boldsymbol{r}(\bar{\tau})-1}\right) .
$$

Note that $\omega^{r}(\rho)$ can be computed by a direct application of Lemma 4.19. 
Lemma 4.23 Let $\boldsymbol{r}$ be such that $Q^{\boldsymbol{r}}$ is lower-acyclic, and consider a segment $\rho$. Let the smoothness distribution $\mathrm{s}$ be defined as follows:

$$
s(\tau)= \begin{cases}r(\tau) & \text { for } \tau \notin \rho, \\ r & \text { otherwise, }\end{cases}
$$

where $r \leqslant \boldsymbol{r}(\tau)$ for all for all $\tau \in \rho, r \in \mathbb{Z}_{\geqslant-1}$. If either one of the following two requirements is satisfied:

(a) $\rho \subsetneq \rho^{\prime}$ for some segment $\rho^{\prime}$, and $\boldsymbol{s}(\tau) \leqslant r$ for all $\tau \in \rho^{\prime}$,

(b) $\omega^{s}(\rho)=m_{\rho}+1$,

then $Q^{s}$ is also lower-acyclic.

Remark 4.24 Since the weight of a segment $\rho$ depends only on the smoothness of edges transversal to $\rho$, we always have $\omega^{\boldsymbol{s}}(\rho)=\omega^{\boldsymbol{r}}(\rho)$.

Proof Using Lemma 3.4, we can study the lower-acyclicity of $Q^{s}$ by studying $H_{0}$ $\left(\mathcal{J}^{s} / \mathcal{J}^{r}\right)$ on the segment $\rho$. This is essentially a one-dimensional problem. Consider then the horizontal segment $\rho$ as shown below; the proof for vertical segments is analogous.

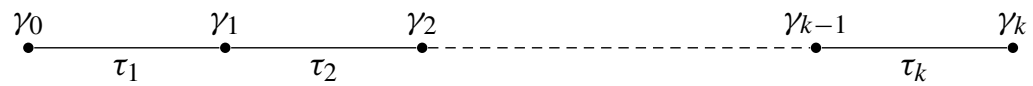

The segment $\rho$ contains the edges $\tau_{1}, \ldots, \tau_{k} \in \stackrel{\circ}{\mathcal{T}}_{1}$, and vertices $\gamma_{0}, \gamma_{1}, \ldots, \gamma_{k}$ form the boundaries of these edges. By definition, $\rho$ contains at least one edge, i.e., $k \geqslant 1$. Moreover, $m_{\tau}=m_{\tau^{\prime}}=: m_{\rho}$ for all edges $\tau, \tau^{\prime} \in \rho$. Let $T$ be the set from Definition 4.22, i.e., the set containing all vertical edges that intersect $L_{\rho}$. Note that $\mathcal{J}^{s} / \mathcal{J}^{r}$ is not supported on any $\bar{\tau} \in T$.

When condition (a) is satisfied, the proof is very simple for the following reason. Firstly, since $\rho \subsetneq \rho^{\prime}$, we must have $m_{\rho}=m_{\rho^{\prime}}$ from the definition of segments. Without loss of generality, let $\gamma_{0} \in \stackrel{\circ}{L}_{\rho^{\prime}} \cap \partial L_{\rho}$. Then, $\mathfrak{I}_{\gamma_{0}}^{r}=\mathfrak{I}_{\gamma_{0}}^{s}$ and, as a consequence, $\mathcal{J}^{s} / \mathcal{J}^{r}$ is not supported on $\gamma_{0}$. Every element of $\mathfrak{I}_{\gamma_{i}}^{s}$ can be expressed as a sum of elements of $\mathfrak{I}_{\bar{\tau}}^{s}, \gamma_{i} \in \bar{\tau} \in T$, and $\mathfrak{I}_{\gamma_{0}}^{s}$. As a result, $H_{0}\left(\mathcal{J}^{s} / \mathcal{J}^{r}\right)$ vanishes.

Let us now examine condition (b). Let $\ell_{\rho}$ be a non-zero linear polynomial that vanishes on $L_{\rho}$. By definition, for all $\tau \in \rho$,

$$
\mathfrak{I}_{\tau}^{s}=\left\{\ell_{\rho}^{r+1} f: f \in \mathcal{P}_{m_{\rho}\left(m_{\rho}-r-1\right)}\right\}=: \mathfrak{I}_{\rho}^{S} .
$$

Let $\ell_{\bar{\tau}}$ be a non-zero linear polynomial that vanishes on vertical edge $\bar{\tau} \in T$. Since $\omega^{s}(\rho)=m_{\rho}+1$, we can use Corollary 4.20 to write:

$$
\mathfrak{I}_{\rho}^{s}=\ell_{\rho}^{r+1} \sum_{\bar{\tau} \in T} \ell_{\bar{\tau}}^{\boldsymbol{s}(\bar{\tau})+1} \mathcal{P}_{\left(m_{\rho}-s(\bar{\tau})-1\right)\left(m_{\rho}-r-1\right)} .
$$

Then, for any $i$, any element of $\mathfrak{I}_{\gamma_{i}}^{s}$ can be written as the sum of elements of $\mathfrak{I}_{\bar{\tau}}^{s}$, $\bar{\tau} \in T$. Since $\mathcal{J}^{s} / \mathcal{J}^{r}$ is not supported on any $\bar{\tau}, H_{0}\left(\mathcal{J}^{s} / \mathcal{J}^{r}\right)=0$.

For both conditions (a) and (b), the claim of lower-acyclicity of $Q^{s}$ follows from the above and Corollary 3.3. 
Lemma 4.23 discusses the setting when the smoothness is reduced across a single segment of the mesh. Its successive applications can help us compute the dimension of a large class of splines on $\mathcal{T}$ with mixed smoothness. The next result is immediate and is completely analogous to Lemma 4.3 which was shown for triangulations. Its statement is simple: if an edge intersects another edge, and the order of smoothness across the latter is -1 , then we can also reduce the order of smoothness across the former to -1 while preserving lower-acyclicity.

Lemma 4.25 Let $\boldsymbol{r}$ be such that $Q^{\boldsymbol{r}}$ is lower-acyclic, and consider an interior edge $\tau \in \stackrel{\circ}{\mathcal{T}}_{1}$. If $\tau^{\prime}$ is another edge such that $\tau \cap \tau^{\prime}$ is not empty, $\boldsymbol{r}\left(\tau^{\prime}\right)=-1$, and $m_{\tau^{\prime}} \geqslant m_{\tau}$, then $Q^{s}$ is lower-acyclic where

$$
\boldsymbol{s}\left(\tau^{\prime \prime}\right)= \begin{cases}\boldsymbol{r}\left(\tau^{\prime \prime}\right) & \tau^{\prime \prime} \neq \tau, \\ -1 & \text { otherwise. }\end{cases}
$$

We are now in a position to present examples where we compute the dimensions of spline spaces on T-meshes that contain holes. The approach will be exactly analogous to the one taken in the previous sub-section, i.e., we will try to see if the spline space can be interpreted as a pruned spline space on a T-mesh without holes. We start by reproducing, and expanding upon, an example from [16].

Example 4.26 ( $C^{1}$ bi-quadratic splines; comparison with Zeng et al. [16]) Consider the problem of building $C^{1}$ bi-quadratic splines on the two domains shown in Figs. 5(a) and (b). Proceeding as in the case of triangulations, we will compute the dimension of such spline spaces by interpreting them as pruned spline spaces on the mesh shown in panel Fig. 5(c).

The dimension of splines on the mesh in Fig. 5(c) can be computed to be 37 using the results from [13]. Then, Lemma 4.23 allows us to see that, for the mesh in Fig. 5(c), we can reduce the smoothness across any segment that is composed of at least two edges. In particular, a combination of Lemmas 4.23 and 4.25 allows us to reduce the smoothness across all dashed edges in Fig. 5(d) and (e) to -1 . The dimensions of the resulting spline spaces are 117 and 85. Then, use of Theorem 4.2 yields the dimension of splines on the meshes in Fig. 5(a) and (b), respectively, as 36 and 40; c.f. the supplementary M2 scripts provided with this paper.

This example borrows from, and expands upon, Example 5.16 from [16]. There, the authors used Bernstein-Bézier methods and computed the dimension of the spline space in Fig. 5(a). First, they compute the dimension for Fig. 5(c); they call the $3 \times 3$ block of extra elements a filler T-mesh. Next, they compute the dimension of splines with zero boundary conditions on the filler T-mesh, i.e., the dimension of bi-quadratic splines that vanish up to order 1 on the boundary of the filler T-mesh. The dimension for Fig. 5(a) is then obtained by subtracting the latter from the former.

Compared to the results here, the applicability of the approach of [16] is constrained by specific choices of filler meshes; for instance, their approach will not help obtain the dimension in Fig. 5(b) by studying Fig. 5(c). Moreover, here we circumvent a particular difficulty that is built into their method: computing the dimension of splines with zero boundary conditions on arbitrarily shaped filler T-meshes. 


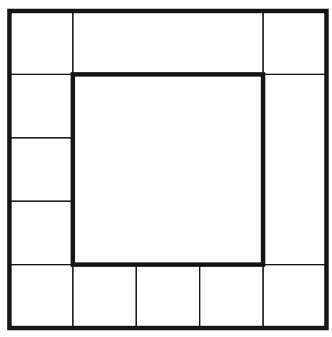

(a)

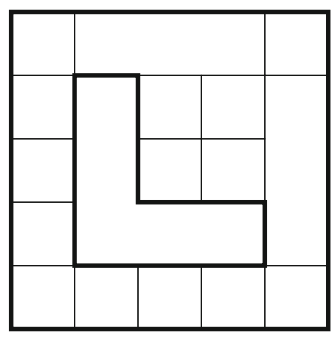

(b)

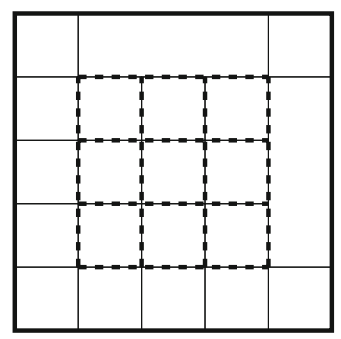

(d)

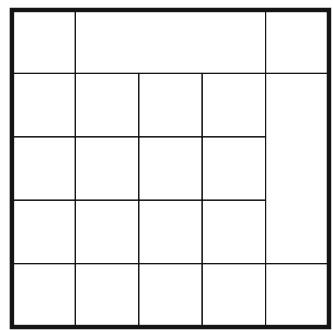

(c)

Fig. 5 Example 4.26 shows how the dimension of bi-quadratic $C^{1}$ splines on the above meshes in a and b can be computed. All boundaries of the respective domains have been displayed in bold; as is clear, the domains are not simply connected. Both of these meshes can be interpreted as pruned meshes obtained from the T-mesh in c. Thus, after computing the dimension on the mesh in c using the results from [13], we can use Corollary 3.3 to compute the dimension when smoothness across the dashed edges in $\mathbf{d}$ and $\mathbf{e}$ has been reduced to $s(\tau)=-1$. This in turn yields the spline space dimension for $\mathbf{a}$ and $\mathbf{b}$

Remark 4.27 Upon visual inspection, the reason for the changes in dimension when going from the mesh in Figs. 5(c) to 5(a) or 5(b) become clear.

- In Fig. 5(a), we have cut out a block of $3 \times 3$ elements; this is exactly the support of one $C^{1}$ bi-quadratic $\mathrm{B}$-spline. The reduction in the dimension by one reflects this observation.

- Similarly, in Fig. 5(b), consider the lower-left $4 \times 4$ block of elements; there are four $C^{1}$ bi-quadratic $\mathrm{B}$-splines supported on these elements. Then, cutting out the L-shaped block of elements splits the supports of three of those B-splines into two disconnected components each. The restrictions of those B-splines to the disconnected components are independent splines themselves; they are not related by any smoothness constraints. As a result, 3 B-splines split up into 6 linearly independent splines, and the dimension count reflects this jump.

Example 4.28 ( $C^{1}$ splines on domains with holes) Consider the problem of building $C^{1}$ splines on the two domains shown in Fig. 6(a) and 6(d). On the mesh in Fig. 6(a), we are interested in splines that are biquadratic polynomials when restricted to any mesh face. On the mesh in Fig. 6(d), on the other hand, we are interested in splines that are biquadratic polynomials restricted to the white faces, and bicubic polynomials when restricted to the blue faces. Proceeding as in the case of triangulations, 


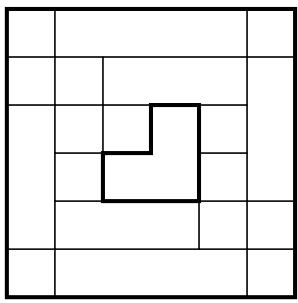

(a)

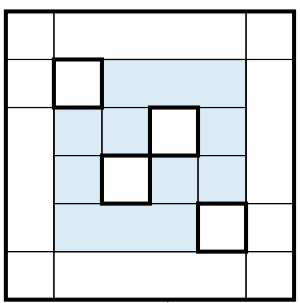

(d)

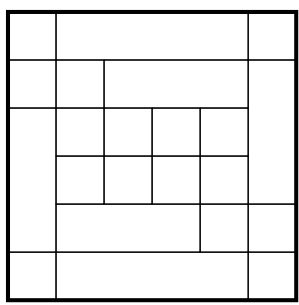

(b)

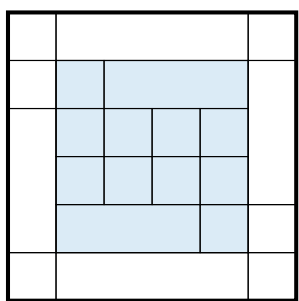

(e)

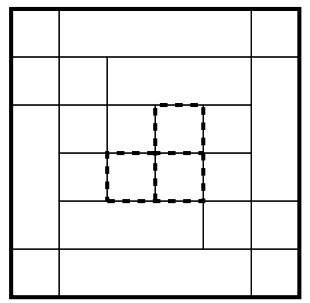

(c)

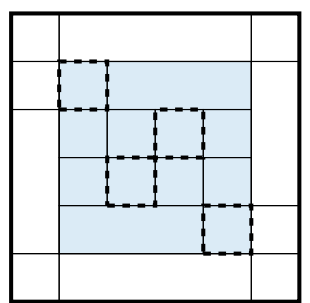

(f)

Fig. 6 Example 4.28 shows how the dimension of $C^{1}$ splines on the above meshes in a and $\mathbf{d}$ can be computed. All boundaries of the respective domains have been displayed in bold; as is clear, the domains are not simply connected. On both meshes, we are interested in splines whose pieces are bi-quadratic polynomials on the faces without color, and bi-cubic polynomials otherwise. First, we use the results from [13] and [14] to get the dimension of splines on meshes in $\mathbf{b}$ and $\mathbf{e}$, respectively. Thereafter, we use Corollary 3.3 to compute the dimension when smoothness across the dashed edges in $\mathbf{c}$ and $\mathbf{f}$ has been reduced to $s(\tau)=-1$. This decouples the faces inside the dashed region from those outside the dashed region. Then, the required dimension can be computed using Theorem 4.2, i.e., by subtracting $\operatorname{dim}\left(\mathcal{P}_{\sigma}\right)$ for each $\sigma$ contained inside the dashed region

we will compute the dimension of such spline spaces by interpreting them as pruned spline spaces on the meshes in Fig. 6(b) and Fig. 6(e).

The dimension of splines on the mesh in Fig. 6(b) can be computed using the results from [13], while the dimension on the mesh in Fig. 6(e) can be computed using [14]. Both are computed to be 30 and 50, respectively. Then, Lemma 4.23 allows us to see that, for the mesh in Fig. 6(b), we can reduce the smoothness across any segment that is composed of at least two edges. For the mesh in Fig. 6(e), Lemma 4.23 allows us to reduce the smoothness across any edge of the mesh. Then, a combination of Lemma 4.23 and 4.25 allows us to reduce the smoothness across all dashed edges in Fig. 6(c) and 6(f) to -1 . The dimensions of the resulting spline spaces are 58 and 118. Thereafter, we can use Theorem 4.2 to compute the dimension of splines on the meshes in Fig. 6(a) and 6(b), respectively, as 31 and 54; c.f. the supplementary M2 scripts provided with this paper. (Again, recall Remarks 4.12 and 4.27.)

\section{Conclusions}

Piecewise-polynomial splines are extensively applied in the fields such as computeraided geometric design [1] and numerical analysis [31]. In practice, spline 
Fig. 7 Splines on meshes of arbitrary topologies [19] can be used to build complex geometries such as the one shown above

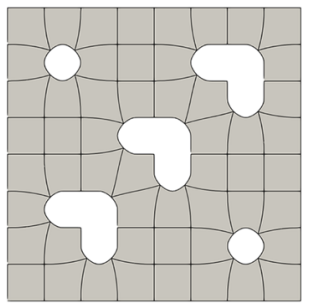

refinements - degree elevation, smoothness reduction, mesh subdivision-are some of the most important operations that yield a richer spline space. These operations are called exact because any spline in the initial spline space can be exactly represented as a member of the refined spline space. The ability to perform these refinements in a local manner is central to efficient applications of splines.

In this paper, we study the problem of dimension computation for splines while focusing on local smoothness reduction. Starting from smooth splines on arbitrary polygonal meshes of $\mathbb{R}^{2}$, we derive results that allow us to compute the exact spline space dimension once smoothness requirements across a subset of the mesh edges have been relaxed. The derived results are very widely applicable, and in order to explore their specific implications we restrict our focus-we show how they can be used to compute the dimension of splines on polygonal meshes, triangulations, and T-meshes of arbitrary topology.

For instance, our results allow us to compute the dimension of splines on T-meshes with holes; see Fig. 6. Such splines can then be used to build smooth surfaces of arbitrary topologies, such as the one shown in Fig. 7-a major application of splines in geometric modelling and numerical analysis [19]. The construction of a splinespace basis that can handle such applications is the focus of ongoing research.

Another particularly interesting research direction is the one opposite to the one we study here-the inexact operation of spline coarsening. These yield subspaces of the original spline space and are also very useful in practice. For example, when numerically solving a PDE, if a complex solution feature simplifies over time, one would want to switch to a subspace of the initial spline space for efficiency. Studying this setting-where a richer spline space is used to compute the dimension of its subspace-will likely require an entirely different approach than the one adopted here.

Open Access This article is licensed under a Creative Commons Attribution 4.0 International License, which permits use, sharing, adaptation, distribution and reproduction in any medium or format, as long as you give appropriate credit to the original author(s) and the source, provide a link to the Creative Commons licence, and indicate if changes were made. The images or other third party material in this article are included in the article's Creative Commons licence, unless indicated otherwise in a credit line to the material. If material is not included in the article's Creative Commons licence and your intended use is not permitted by statutory regulation or exceeds the permitted use, you will need to obtain permission directly from the copyright holder. To view a copy of this licence, visit http://creativecommons.org/licenses/by/4. $0 \%$. 


\section{References}

1. Farin, G.E., Hoschek, J., Kim, M.-S.: Handbook of Computer Aided Geometric Design. Elsevier, Amsterdam (2002)

2. Cottrell, J.A., Hughes, T.J.R., Bazilevs, Y.: Isogeometric Analysis: Toward Integration of CAD and FEA. Wiley, Hoboken (2009)

3. Strang, G.: Piecewise polynomials and the finite element method. Bull. Am. Math. Soc. 79(6), 11281137 (1973)

4. Strang, G.: The dimension of piecewise polynomial spaces, and one-sided approximation. In: Conference on the Numerical Solution of Differential Equations, pp 144-152. Springer (1974)

5. Schumaker, L.L.: Bounds on the dimension of spaces of multivariate piecewise polynomials. Rocky Mt. J. Math. 14(1), 251-264 (1984)

6. Alfeld, P., Schumaker, L.L.: The dimension of bivariate spline spaces of smoothness $r$ for degree $d \geqslant 4 r+1$. Constr. Approx. 3(1), 189-197 (1987)

7. Billera, L.J.: Homology of smooth splines: Generic triangulations and a conjecture of strang. Trans. Am. Math. Soc. 310(1), 325-340 (1988)

8. Schenck, H., Stillman, M.: Local cohomology of bivariate splines. J. Pure Appl. Algebra 117, 535548 (1997)

9. Schenck, H., Stillman, M.: A family of ideals of minimal regularity and the hilbert series of $C^{r}(\Delta)$. Adv. Appl. Math. 19(2), 169-182 (1997)

10. McDonald, T., Schenck, H.: Piecewise polynomials on polyhedral complexes. Adv. Appl. Math. 42(1), 82-93 (2009)

11. DiPasquale, M.: Dimension of mixed splines on polytopal cells. Math. Comput. 87(310), 905-939 (2018)

12. Toshniwal, D., Hughes, T.J.R.: Polynomial splines of non-uniform degree on triangulations: Combinatorial bounds on the dimension. Comput. Aided Geom. Des. 75, 101763 (2019)

13. Mourrain, B.: On the dimension of spline spaces on planar T-meshes. Math. Comput. 83(286), 847871 (2014)

14. Toshniwal, D., Mourrain, B., Hughes, T.J.R.: Advances in Computational Mathematics (accepted). arXiv:1903.05949 [math] https://doi.org/10.1007/s10444-020-09829-4 (2019)

15. Bracco, C., Lyche, T., Manni, C., Roman, F., Speleers, H.: Generalized spline spaces over T-meshes: Dimension formula and locally refined generalized B-splines. Appl. Math. Comput. 272, 187-198 (2016)

16. Zeng, C., Wu, M., Deng, F., Deng, J.: Dimensions of spline spaces over non-rectangular T-meshes. Adv. Comput. Math. 42(6), 1259-1286 (2016)

17. Schenck, H., Sorokina, T.: Subdivision and spline spaces. Constr. Approx. 47(2), 237-247 (2018)

18. Toshniwal, D., Villamizar, N.: Dimension of polynomial splines of mixed smoothness on T-meshes. Comput. Aided Geom. Des. 80, 101880 (2020)

19. Toshniwal, D., Speleers, H., Hughes, T.J.R.: Smooth cubic spline spaces on unstructured quadrilateral meshes with particular emphasis on extraordinary points: Geometric design and isogeometric analysis considerations. Comput. Methods Appl. Mech. Eng. 327, 411-458 (2017)

20. Alfeld, P., Piper, B., Schumaker, L.L.: Spaces of bivariate splines on triangulations with holes. In: Proceedings of China-U.S. Joint Conference on Approximation Theory (Hangzhou, 1985), vol. 3, pp. 1-10 (1987)

21. Jia, R.Q.: Lower bounds on the dimension of spaces of bivariate splines. In: Multivariate Approximation and Interpolation (Duisburg, 1989), vol. 94 of International Series of Numerical Mathematics, pp. 155-165. Birkhäuser, Basel (1990)

22. Chui, C.K.: Multivariate Splines, vol. 54. Siam (1988)

23. Hatcher, A.: Algebraic Topology. Cambridge University Press, Cambridge (2002)

24. Alfeld, P., Schumaker, L.L.: On the dimension of bivariate spline spaces of smoothness $r$ and degree $d=3 r+1$. Numer. Math. 57(1), 651-661 (1990)

25. Schenck, H.: Computational Algebraic Geometry, vol. 58. Cambridge University Press, Cambridge (2003)

26. Hong, D.: Spaces of bivariate spline functions over triangulation. J. Approx. Theory 7(1), 56-75 (1991)

27. Ibrahim, A.K., Schumaker, L.L.: Super spline spaces of smoothness $r$ and degree $d \geqslant 3 r+2$. Constr. Approx. 7(3), 401-423 (1991) 
28. Geramita, A., Schenck, H.K.: Fat points, inverse systems, and piecewise polynomial functions. J. Algebra 204(1), 116-128 (1998)

29. Mourrain, B., Villamizar, N.: Homological techniques for the analysis of the dimension of triangular spline spaces. J. Symb. Comput. 50, 564-577 (2013)

30. Grayson, D.R., Stillman, M.E.: Macaulay2, a software system for research in algebraic geometry. Available at https://faculty.math.illinois.edu/Macaulay2/

31. Hughes, T.J.R., Cottrell, J.A., Bazilevs, Y.: Isogeometric Analysis: CAD, finite elements, NURBS, exact geometry and mesh refinement. Comput. Methods Appl. Mech. Eng. 194, 4135-4195 (2005)

Publisher's note Springer Nature remains neutral with regard to jurisdictional claims in published maps and institutional affiliations. 